This report was prepared as an account of work sponsored by an agency of the United States Government. Neither the United States Government nor any agency thereof, nor any of their employees, makes any warranty, express or implied, or assumes any legal liability or responsibility for the accuracy, completeness, or usefulness of any information, apparatus, product, or process disclosed, or represents that its use would not infringe privately owned rights. Reference herein to any specific commercial product, process, or service by trade name, trademark, manufacturer, or otherwise does not necessarily constitute or imply its endorsement, recommendation, or favoring by the United States Government or any agency thereof. The views and opinions of authors expressed herein do not necessarily state or reflect those of the United States Government or any agency thereof.

\title{
List of Currently Classified Documents Relative to Hanford Operations and of Potential Use in the Columbia River Comprehensive Impact Assessment January 1, 1973 - June 20, 1994
}

Columbia River Comprehensive Impact Assessment Project

T. B. Miley

L. R. Huesties

February 1995

Prepared for the U.S. Department of Energy under Contract DE-AC06-76RLO 1830

Pacific Northwest Laboratory

Richland, Washington 99352 



\section{DISCLAIMER}

Portions of this document may be illegible in electronic image products. Images are produced from the best available original document. 


\section{Preface}

The Columbia River Comprehensive Impact Assessment (CRCIA) Project at the Pacific Northwest Laboratory (PNL) ${ }^{(a)}$ is evaluating the current human and ecological risks from contaminants in the Columbia River. The risks to be studied are those attributable to past and present activities at the Hanford Site. The Hanford Site is located in southcentral Washington State near the town of Richland, Washington. Human risk from exposure to radioactive and hazardous materials will be addressed for a range of river use options. Ecological risk will be evaluated relative to the health of the current river ecosystem. The overall purpose of the project is to determine if enough contamination exists in the Columbia River to warrant cleanup actions under applicable environmental regulations.

This report identifies currently classified documents that 1) are relative to Hanford operations, 2) may contain information relevant to a comprehensive study of the Columbia River, and 3) were generated between January 1, 1973 and June 20, 1994. Three previous reports (Haerer 1990a; Haerer 1990b; HEDR Staff 1993) identify approximately 18,000 classified documents originated at the Hanford Site from 1944-1972 and relative to Hanford production facilities operations. Many of these documents have been declassified since publication of these three previous reports. With the addition of this fourth report, a complete listing of classified documents, which may contain information of potential use to the CRCIA Project, is provided from start up of operations at the Hanford Site to the present.

Historically, the U.S. Department of Energy (DOE) operated nine production reactors (B, C, D, DR, F, H, KE, KW, and N) along the Hanford Reach of the Columbia River. The Hanford Reach extends 85 kilometers (51 miles) downstream from Priest Rapids Dam to the head of the McNary Pool just north of the city of Richland. Eight of these reactors used single-pass cooling systems that released radionuclides, process chemicals (including chemicals that inhibited corrosion), and heated water into the Columbia River. These eight reactors were all shut down by early 1971 . The $N$ reactor, which used a closed-loop primary cooling system, operated between 1963 and 1987. It was deactivated in 1989 and is in the process of being decontaminated and decommissioned. Past operations of Hanford's processing plants also resulted in contaminated effluents, some of which have made their way to the Columbia River through the groundwater. These plants were the bismuth phosphate process plants (B and T Plants), plutonium uranium extraction plant (A Plant/PUREX), reduction and oxidation plant (S Plant/REDOX), and the plutonium finishing plant (Z Plant/PFP).

The CRCIA Project is a joint activity of three government agencies at the Hanford Site: DOE, U.S. Environmental Protection Agency, and Washington State Department of Ecology. These agencies have signed an agreement known officially as the Hanford Federal Facility Agreement and Consent Order and unofficially as the Tri-Party Agreement (TPA) (Ecology et al. 1994). Milestones have been adopted for the TPA that identify actions needed to ensure acceptable progress toward Hanford Site compliance with the Comprehensive Environmental Response, Compensation, and Liability Act of 1980 (CERCLA), the Resource Conservation and Recovery Act of 1976 (RCRA), and the Washington State Hazardous Waste Management Act of 1976 (HWMA). The January 1994 revision to the TPA (Change Order number M-13-93-06) incorporates adjustments made to milestones designed to address cleanup

(a) Pacific Northwest Laboratory is operated for the U.S. Department of Energy by Battelle Memorial Institute. 
strategies and achieve timely remedial decisions and actions concerning the Columbia River. This change order included the new Milestone M-13-80 that established the CRCIA Project.

The environmental quality of the Columbia River is of special interest to the public, government, and tribal governments as a source of drinking water, for crop irrigation, as ecological habitat, and for recreation. The following actions have been taken to encourage public involvement in the CRCIA Project:

- PNL has an open door policy for this project. Non-PNL individuals can visit the laboratory, interact with scientists, and observe work in progress.

- Data and documents used in the CRCIA Project are being made available to all interested parties.

- Public meetings are being conducted to obtain input to the development of work scope and technical approaches as well as to review data and work progress. 


\section{Summary}

The Columbia River Comprehensive Impact Assessment (CRCIA) Project seeks to estimate the current risk from the Columbia River resulting from past and present Hanford activities. To resolve the question of the current risk, it is necessary for the CRCIA Project to have access to any classified information that may be relevant to this study. The purpose of this report is to present the results of the search for relevant classified information. This search was subsequent to the publication of documents applicable to the CRCIA Project in the data compendium (Eslinger et al. 1994).

There are two classified matter control centers operated by two prime contractors at the Hanford Site. One is operated by Pacific Northwest Laboratory (PNL) and the other is operated by Westinghouse Hanford Company (WHC). Only the WHC collection contained information relevant to a study of the Columbia River in the time frame of interest: January 1, 1973 through June 20, 1994.

A list of the classified documents in the WHC collection is maintained in the WHC Classified Document Control database. The WHC Classified Document Control database was searched. The search criteria were the dates of interest and the basic keywords used for the CRCIA Project's data compendium (Eslinger et al. 1994). All Hanford-generated, Hanford-related entries that were applicable to the CRCIA Project and the dates of interest were provided. The resulting list of 477 titles comprises the Appendix of this report. The information given for each title is exactly as it appears in the database. Any inconsistencies are the result of duplicating the database. 


\section{Glossary}

\begin{tabular}{|c|c|}
\hline AEC-RL & Atomic Energy Commission, Richland, Washington \\
\hline ARHCO & Atlantic Richfield Hanford Company \\
\hline CERCLA & $\begin{array}{l}\text { Comprehensive Environmental Response, Compensation, and Liability } \\
\text { Act of } 1980\end{array}$ \\
\hline CRCIA & Columbia River Comprehensive Impact Assessment \\
\hline DOE & U.S. Department of Energy \\
\hline DOE-HQ & U.S. Department of Energy, Headquarters \\
\hline DOE-RL & U.S. Department of Energy, Richland Operations Office \\
\hline HEDL-RL & Hanford Engineering Development Laboratory, Richland, Washington \\
\hline HEDR & Hanford Environmental Dose Reconstruction Project \\
\hline HWMA & Washington State Hazardous Waste Management Act of 1976 \\
\hline ISOCHEM-RL & ISOCHEM Incorporated, Richland, Washington \\
\hline PNL & Pacific Northwest Laboratory \\
\hline RCRA & Resource Conservation and Recovery Act of 1976 \\
\hline $\mathrm{RHO}$ & Rockwell Hanford Operations \\
\hline RHO-RL & Rockwell Hanford Operations, Richland, Washington \\
\hline TPA & $\begin{array}{l}\text { Tri-Party. Agreement } \\
\text { Hanford Federal Facility Agreement and Consent Order }\end{array}$ \\
\hline UNC & United Nuclear Corporation \\
\hline UNC-RL & United Nuclear Corporation, Richland, Washington \\
\hline WHC & Westinghouse Hanford Company \\
\hline WHC-RL & Westinghouse Hanford Company \\
\hline
\end{tabular}

vii 


\section{Contents}

Preface $\ldots \ldots \ldots \ldots \ldots \ldots \ldots \ldots \ldots \ldots \ldots \ldots \ldots \ldots \ldots \ldots \ldots \ldots \ldots$

Summary $\ldots \ldots \ldots \ldots \ldots \ldots \ldots \ldots \ldots \ldots \ldots \ldots \ldots \ldots \ldots \ldots \ldots \ldots$

Glossary $\ldots \ldots \ldots \ldots \ldots \ldots \ldots \ldots \ldots \ldots \ldots \ldots \ldots \ldots \ldots \ldots \ldots \ldots$

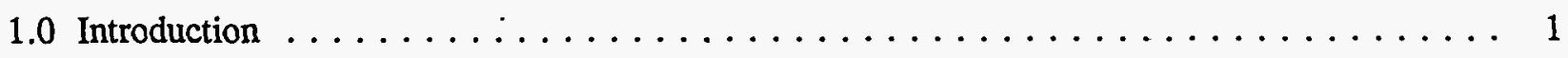

1.1 Background $\ldots \ldots \ldots \ldots \ldots \ldots \ldots \ldots \ldots \ldots \ldots \ldots \ldots \ldots \ldots$

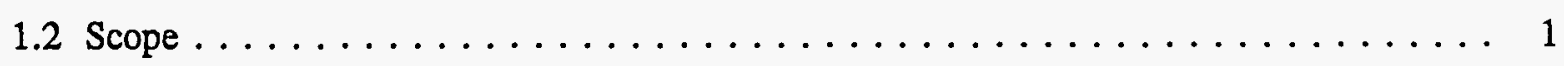

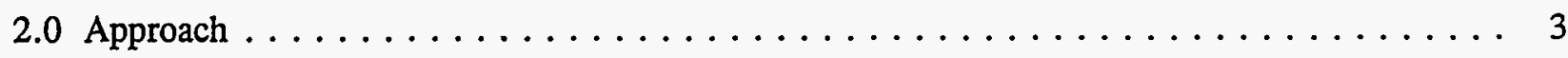

2.1 Identification of Document Sources $\ldots \ldots \ldots \ldots \ldots \ldots \ldots \ldots \ldots \ldots \ldots \ldots$

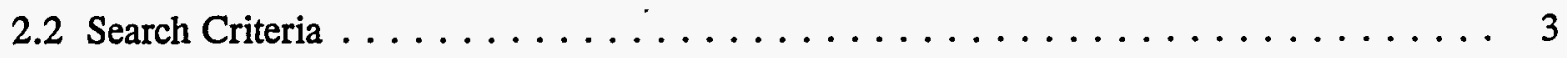

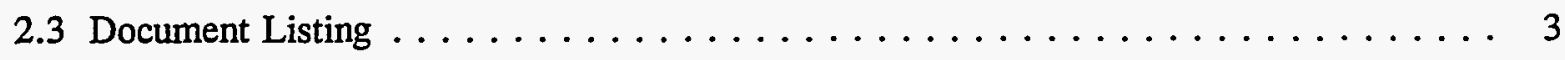

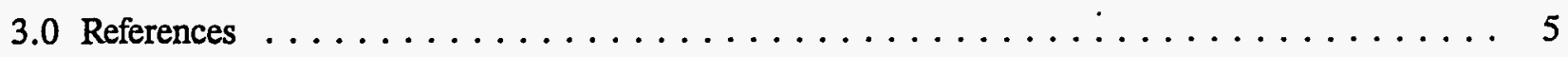

Appendix - Classified Document Titles $\ldots \ldots \ldots \ldots \ldots \ldots \ldots \ldots \ldots \ldots$ 


\subsection{Introduction}

\subsection{Background}

Data relevant to the Columbia River environment have been collected since the start-up of Hanford operations. The data were recorded in documents that were originated as classified, especially during production operations. Though most of the information in these documents is no longer classified, declassification of the documents themselves has historically been done by request only. There has only recently been a major effort to declassify most Hanford originated documents.

\subsection{Scope}

The scope of this report is to identify currently classified documents that 1) are relative to Hanford operations, 2) may contain information relevant to a comprehensive study of the Columbia River, and 3) were generated between January 1, 1973 and June 20,1994. The report follows three previous reports of classified documents relative to Hanford production facilities operations. Haerer (1990a, $1990 \mathrm{~b}$ ) contain titles of classified documents originated at the Hanford Site from the start up of Hanford operations (1944) through December 31, 1960. HEDR Staff (1993) contains titles of classified documents originated at the Hanford Site from January 1, 1961 through December 31, 1972. The set of four reports will represent a complete search for classified documents, which may contain information of potential use to the CRCIA Project, from start up through June 20, 1994.

Other reports relative to this report are Eslinger et al. (1994), Gydesen (1992), and Hall (1991). Whereas Eslinger et al. (1994) reported the results of a variety of database searches for documents pertinent to the Columbia River Comprehensive Impact Assessment Project, the Westinghouse Hanford Company (WHC) Classified Document Control database had not been searched by the time of publication. This report documents the results of that search. Gydesen (1992) identifies the declassifications of the documents in Haerer (1990a, 1990b), and Hall (1991) provides references for radionuclide releases to the Columbia River. 


\subsection{Approach}

\subsection{Identification of Document Sources}

There are two classified matter control centers operated by two prime contractors at the Hanford Site. Documents under the custodianship of the Pacific Northwest Laboratory (PNL) are held at the PNL Classified Matter Control Center, located in the Hanford Technical Library building. Documents under the custodianship of Westinghouse Hanford Company (WHC) are held at WHC Classified Document Control in the Federal Building and at the Records Holding Area. The PNL holdings were found to contain no currently classified, Hanford generated, Hanford related reports for the time period of interest. Only the WHC collection contained information relevant to a study of the Columbia River in the time frame of interest: January 1, 1973 through June 20, 1994.

\subsection{Search Criteria}

A list of the classified documents in the WHC collection is maintained in the WHC Classified Document Control database. A search of the WHC Classified Document Control database was conducted on June 20,1994, utilizing two search criteria. The first was that the date for the document was between January 1, 1973 and the date of the search, June 20,1994. The second criterion was that the document was both Hanford-generated and Hanford-related. Keywords were used to identify documents that fit the second criterion. The keywords used were the same as those used for the data compendium (Eslinger et al. 1994).

\subsection{Document Listing}

The document listing of 477 titles in the Appendix reflects those in the WHC Classified Document Control database which met the search criteria described above. The information given for each title is exactly as it appears in the database. Any inconsistencies are the result of duplicating the database.

An ongoing declassification effort has been taking place under the Secretary of Energy's new policy. of openness. Some of the documents listed here may have been declassified since June 20, 1994 when the database was searched. 
... 


\subsection{References}

Ecology - Washington State Department of Ecology, U.S. Environmental Protection Agency, and U.S. Department of Energy. 1994. Hanford Federal Facility Agreement and Consent Order.

Document No. 89-10, Rev. 3 (The Tri-Party Agreement), Washington State Department of Ecology,

Olympia, Washington.

Eslinger, P. W., L. R. Huesties, A. D. Maughan, T. B. Miley, and W. H. Walters. 1994. Data Compendium for the Columbia River Comprehensive Impact Assessment. PNL-9785, Pacific Northwest Laboratory, Richland, Washington.

Gydesen, S. P. 1992. Declassifications Requested by the Technical Steering Panel of Hanford Documents Produced 1944-1960. PNWD-2024 HEDR, Battelle, Pacific Northwest Laboratories, Richland, Washington.

Haerer, H. A. 1990a. Hanford Site Originated Documents, 1944-1960, Secret and Confidential. PNL-10238, Pacific Northwest Laboratory, Richland, Washington.

Haerer, H. A. 1990b. Hanford Site Originated Documents, 19441960, Secret and Confidential. PNL-10238 Add. 1, Pacific Northwest Laboratory, Richland, Washington.

Hall, R. B. 1991. Letter Report: References for Radioactive Releases to the Columbia River from Hanford Operations, 1944-1957. PNL-7869 HEDR, Pacific Northwest Laboratory, Richland, Washington.

HEDR Staff. 1993. List of Currently 'Classified Documents Relative to Hanford Production Facilities Operations Originated on the Hanford Site Between 1961 and 1972. PNWD-2129 HEDR. Battelle, Pacific Northwest Laboratories, Richland, Washington. 
... 


\section{Appendix}

\section{Classified Document Titles}




\section{Appendix}

\section{Classified Document Titles}

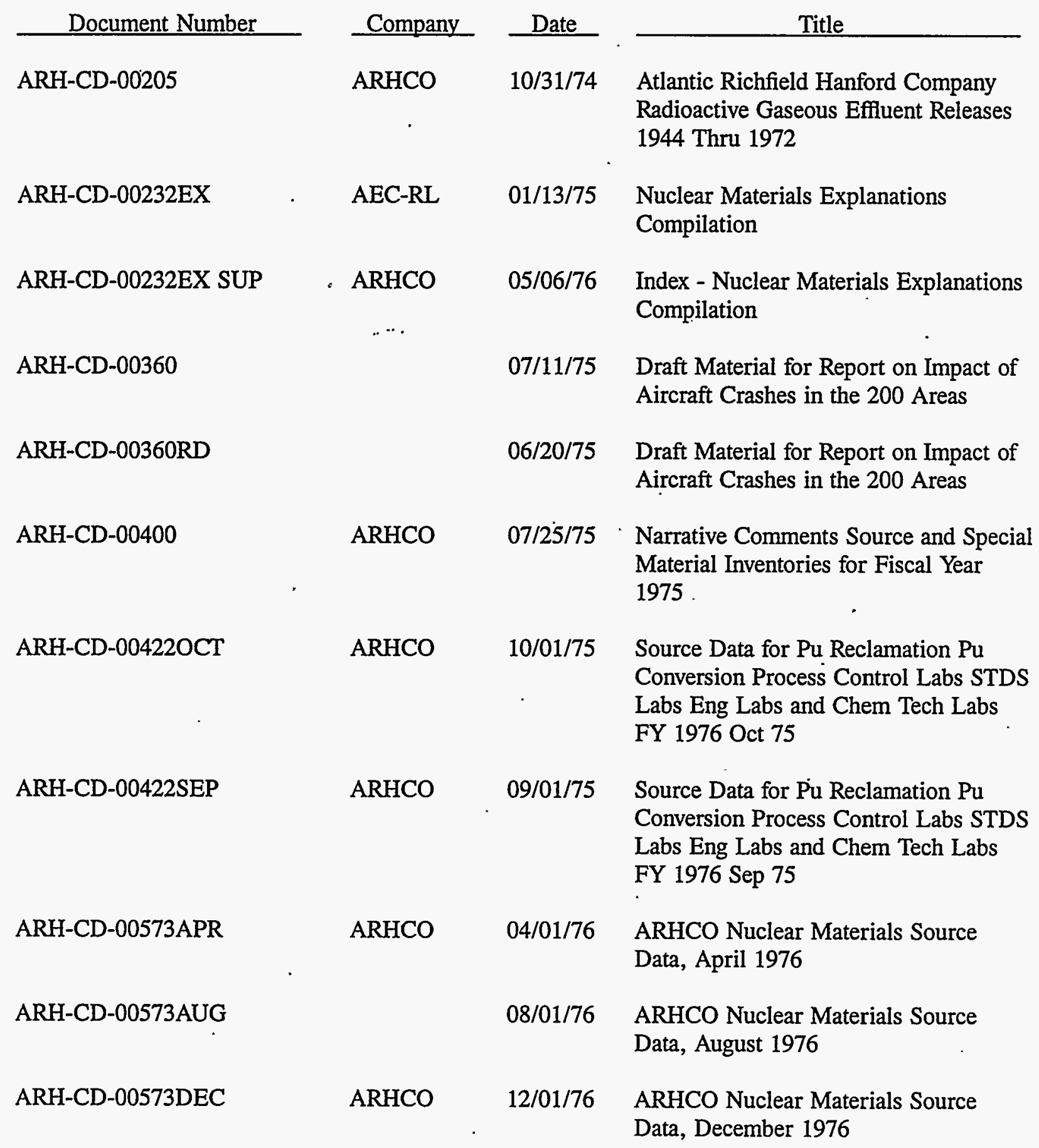


Document Number

ARH-CD-00573FEB

ARH-CD-00573JAN

ARH-CD-00573JUL

ARH-CD-00573JUN

ARH-CD-00573MAR

ARH-CD-00573MAY ... .

ARH-CD-00573NOV

ARH-CD-005730CT

ARH-CD-00573SEP

ARH-CD-00818

ARH-CD-00874APR

ARH-CD-00874FEB

ARH-CD-00874JAN

ARH-CD-00874JUN

ARH-CD-00874MAR

$\frac{\text { Company }}{02 / 01 / 76}$

ARHCO 01/01/76

ARHCO

07/01/76

ARHCO

ARHCO

03/01/76

06/01/76

ARHCO Nuclear Materials Source Data, February 1976

ARHCO Nuclear Materials Source Data, January 1976

ARHCO Nuclear Materials Source Data, July 1976

ARHCO Nuclear Materials Source Data, June 1976

ARHCO Nuclear Materials Source Data, March 1976

05/01/76 ARHCO Nuclear Materials Source Data, May 1976

11/01/76 ARHCO Nuclear Materials Source Data, November 1976

10/01/76 ARHCO Nuclear Materials Source Data, October 1976

ARHCO 09/01/76

ARHCO Nuclear Materials Source Data, September 1976

ARHCO 10/26/76 Narrative Comments

Source and Special Material Inventories for FY 1976

ARHCO 04/01/77 ARHCO Nuclear Materials Source Data, April 1977

02/01/77, ARHCO Nuclear Materials Source Data, February 1977

ARHCO $\quad 01 / 01 / 77$

ARHCO Nuclear Materials Source Data, January 1, 1977

ARHCO 06/01/77 Nuclear Materials Source Data June 1977

ARHCO 03/01/77
ARHCO Nuclear Materials Source Data, March 1977 


\begin{tabular}{|c|c|c|c|}
\hline Document Number & Company & Date & Title \\
\hline ARH-CD-00874MAY & ARHCO & $05 / 01 / 77$ & $\begin{array}{l}\text { ARHCO Nuclear Materials Source } \\
\text { Data, May } 1977\end{array}$ \\
\hline ARH-CD-01018 & $\begin{array}{c}\text { ARHCO } \\
;\end{array}$ & $12 / 09 / 74$ & $\begin{array}{l}\text { Misdirected Shipment of Radioactive } \\
\text { Material }\end{array}$ \\
\hline ARH-N-00073 & $\begin{array}{l}\text { ISOCHEM } \\
\text {-RL }\end{array}$ & $04 / 21 / 77$ & $\begin{array}{l}\text { Personal Notes } \\
\text { Chemical Processing Design } \\
\text { Engineering }\end{array}$ \\
\hline ARH-02498APR & ARHCO & $04 / 01 / 73$ & $\begin{array}{l}\text { Plutonium Processing Source } \\
\text { Data - FY 1973, April } 1973\end{array}$ \\
\hline ARH-02498FEB & ARHCO & $02 / 01 / 73$ & $\begin{array}{l}\text { Plutonium Processing Source } \\
\text { Data - FY 1973, February } 1973\end{array}$ \\
\hline ARH-02498JAN & ARHCO & $01 / 01 / 73$ & $\begin{array}{l}\text { Plutonium Processing Source } \\
\text { Data - FY 1973, January } 1973\end{array}$ \\
\hline ARH-02498JUN & ARHCO & $06 / 01 / 73$ & $\begin{array}{l}\text { Plutonium Processing Source } \\
\text { Data - FY 1973, June } 1973\end{array}$ \\
\hline ARH-02498MAR & ARHCO & $03 / 01 / 73$ & $\begin{array}{l}\text { Plutonium Processing Source } \\
\text { Data - FY 1973, March } 1973\end{array}$ \\
\hline ARH-02498MAY. & ARHCO & $05 / 01 / 73$ & $\begin{array}{l}\text { Plutonium Processing Source } \\
\text { Data - FY 1973, May } 1973 .\end{array}$ \\
\hline ARH-02499APR & ARHCO & $04 / 01 / 73$ & $\begin{array}{l}\text { Plutonium Reclamation Source } \\
\text { Data - FY 1973, April } 1973\end{array}$ \\
\hline ARH-02499FEB & ARHCO & $02 / 01 / 73$ & $\begin{array}{l}\text { Plutonium Reclamation Source } \\
\text { Data - FY 1973, February } 1973\end{array}$ \\
\hline ARH-02499JAN & ARHCO & $01 / 01 / 73$ & $\begin{array}{l}\text { Plutonium Reclamation Source } \\
\text { Data - FY 1973, January } 1973\end{array}$ \\
\hline ARH-02499JUN & ARHCO & $06 / 01 / 73$ & $\begin{array}{l}\text { Plutonium Reclamation Source } \\
\text { Data - FY 1973, June } 1973\end{array}$ \\
\hline ARH-02499MAR & ARHCO & $03 / 01 / 73$ & $\begin{array}{l}\text { Plutonium Reclamation Source } \\
\text { Data - FY 1973, March } 1973\end{array}$ \\
\hline ARH-02499MAY. & ARHCO & $05 / 01 / 73$ & $\begin{array}{l}\text { Plutonium Reclamation Source } \\
\text { Data - FY 1973, May } 1973\end{array}$ \\
\hline
\end{tabular}


Document Number

ARH-025043

ARH-025044

ARH-02846AUG

ARH-02846DEC

ARH-02846JAN

ARH-02846JUL

ARH-02846NOV

ARH-028460CT

ARH-02846SEPT

H-3-57531 SH1 RO A

H-3-57531 SH1 RO B

H-3-57531 SH1 R1 M

HEDL-P-PAR-137

HEDL-810152
Company Date

ARHCO 01/01/73

$04 / 01 / 73$

Burial Garden Records - VVA FY 1973 Month End and Source Data - January Thru March 1973

Burial Garden Records - VVA FY 1973 Month End and Source Data April Thru June 1973

ARHCO 08/01/73 Plutonium Processing Source Data - FY 1974, August 1973

ARHCO

$12 / 01 / 73$

Plutonium Processing Source

Data - FY 1974, December 1973

ARHCO

$01 / 01 / 74$

Plutonium Processing Source

Data - FY 1974, January 1974

ARHCO 07/01/73 Plutonium Processing Source

Data - FY 1974, July 1973

ARHCO 11/01/73 Plutonium Processing Source

Data - FY 1974, November 1973

ARHCO

$10 / 01 / 73$

Plutonium Processing Source

Data - FY 1974, October 1973

ARHCO

$09 / 01 / 73$

Plutonium Processing Source

Data - FY 1974, September 1973

WHC-RL 07/13/90 Security Power Sources One Line Diagram

WHC-RL 07/13/90 Security Power Sources One Line Diagram

WHC-RL 07/13/90 Security Power Sources One Line Diagram

HEDL-RL 01/13/86 Materials Produced from Foreign Sources

UNC-RL $\quad 01 / 29 / 82$
A Compilation of Notes Generated and Received for 2020 Study 


\begin{tabular}{|c|c|c|c|}
\hline Document Number & Company & Date & Title \\
\hline HEDL-810419 & HEDL-RL & $12 / 11 / 84$ & $\begin{array}{l}\text { Contingency Plan Changes and Security } \\
\text { Upgrade Impact } 300-400 \text { Areas }\end{array}$ \\
\hline HEDL-810525 & & $10 / 18 / 85$ & $\begin{array}{l}\text { Security System Upgrades (Impact } \\
\text { Statements) }\end{array}$ \\
\hline HEDL-810526 & HEDL-RL & $10 / 23 / 85$ & $\begin{array}{l}\text { Results of Analyses of Purex D-5 and } \\
\text { A-3 Tank Samples to Characterize Key } \\
14011 \text { and Key } 14306 \text { N-Reactor Spent } \\
\text { Fuel Batches }\end{array}$ \\
\hline HEDL-810550 & & $04 / 01 / 86$ & $\begin{array}{l}\text { Solid State Track Recorder Source Term } \\
\text { Measurements at the Little Boy Replica }\end{array}$ \\
\hline HEDL-810619 & HEDL-RL & $12 / 15 / 86$ & $\begin{array}{l}\text { Results of Analyses of Purex D5 Tank } \\
\text { Samples and Composites for } \\
\text { Characterization of N Reactor MK-IV } \\
\text { Spent Fuel Batch } 14832\end{array}$ \\
\hline HEDL-810683 & & $06 / 15 / 87$ & SNM Detector Test Source Composition \\
\hline ISO-01012 & ARCHO & $12 / 27 / 88$ & $\begin{array}{l}\text { Chemical Analysis of Drivers from } 2.1 \\
\text { Coproduct Block (PT-66) }\end{array}$ \\
\hline RHO-CD-00061 & . & $07 / 29 / 78$ & Preparation of Round Robin Samples \\
\hline RHO-CD-00061RD & & $07 / 09 / 77$ & Preparation of Round Robin Samples \\
\hline RHO-CD-00149 & & $10 / 26 / 77$ & $\begin{array}{l}\text { Narrative Comments: Source and } \\
\text { Special Material Inventories for } \\
\text { Fiscal Year } 1977\end{array}$ \\
\hline RHO-CD-77-0005110 & RHO-RL & 10/01/77 & $\begin{array}{l}\text { Rockwell Nuclear Materials Source } \\
\text { Data, October } 1977\end{array}$ \\
\hline RHO-CD-77-0005111 & RHO-RL & $11 / 01 / 77$ & $\begin{array}{l}\text { Rockwell Nuclear Materials Source } \\
\text { Data, November } 1977\end{array}$ \\
\hline RHO-CD-77-0005112 & RHO-RL & $12 / 01 / 77$ & $\begin{array}{l}\text { Rockwell Nuclear Materials Source } \\
\text { Data, December } 1977\end{array}$ \\
\hline RHO-CD-77-000517 & & $06 / 01 / 77$ & $\begin{array}{l}\text { Rockwell Nuclear Materials Source } \\
\text { Data, July } 1977\end{array}$ \\
\hline
\end{tabular}




\begin{tabular}{|c|c|c|c|}
\hline Document Number & Company & Date & 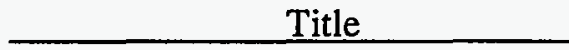 \\
\hline RHO-CD-77-000518 & & $08 / 01 / 77$ & $\begin{array}{l}\text { Rockwell Nuclear Materials Source } \\
\text { Data, August } 1977\end{array}$ \\
\hline RHO-CD-77-000519 & & 09/01/77 & $\begin{array}{l}\text { Rockwell Nuclear Materials Source } \\
\text { Data, September } 1977\end{array}$ \\
\hline RHO-CD-77-000519 VOL2 & & 09/01/77 & $\begin{array}{l}\text { Rockwell Nuclear Materials Source } \\
\text { Data, September } 1977\end{array}$ \\
\hline RHO-CD-78-000511 & RHO-RL & $01 / 01 / 78$ & $\begin{array}{l}\text { Rockwell Nuclear Materials Source } \\
\text { Data, January } 1978\end{array}$ \\
\hline RHO-CD-78-0005110 & & $10 / 01 / 78$ & $\begin{array}{l}\text { Rockwell Nuclear Materials Source } \\
\text { Data, October } 1978\end{array}$ \\
\hline RHO-CD-78-0005111 & RHO-RL & $11 / 01 / 78$ & $\begin{array}{l}\text { Rockwell Nuclear Materials Source } \\
\text { Data, November } 1978\end{array}$ \\
\hline RHO-CD-78-0005112 & RHO-RL & $12 / 01 / 78$ & $\begin{array}{l}\text { Rockwell Nuclear Materials Source } \\
\text { Data, December } 1978\end{array}$ \\
\hline RHO-CD-78-000512 BK1 & RHO-RL & $02 / 01 / 78$ & $\begin{array}{l}\text { Rockwell Nuclear Materials Source } \\
\text { Data, February } 1978\end{array}$ \\
\hline RHO-CD-78-000513 & RHO-RL & $03 / 01 / 78$ & $\begin{array}{l}\text { Rockwell Nuclear Materials Source } \\
\text { Data, March } 1978\end{array}$ \\
\hline RHO-CD-78-000514 & RHO-RL & 04/01/78 & $\begin{array}{l}\text { Rockwell Nuclear Materials Source } \\
\text { Data, April } 1978\end{array}$ \\
\hline RHO-CD-78-000515 & RHO-RL & $05 / 01 / 78$ & $\begin{array}{l}\text { Rockwell Nuclear Materials Source } \\
\text { Data, May } 1978\end{array}$ \\
\hline RHO-CD-78-000516 & RHO-RL & $06 / 01 / 78$ & $\begin{array}{l}\text { Rockwell Nuclear Materials Source } \\
\text { Data, June } 1978\end{array}$ \\
\hline RHO-CD-78-000517 & RHO-RL & $07 / 01 / 78$ & $\begin{array}{l}\text { Rockwell Nuclear Materials Source } \\
\text { Data, July } 1978\end{array}$ \\
\hline RHO-CD-78-000518 & RHO-RL & $08 / 01 / 78$ & $\begin{array}{l}\text { Rockwell Nuclear Materials Source } \\
\text { Data, August } 1978\end{array}$ \\
\hline RHO-CD-78-000519 & RHO-RL & 09/01/78 & $\begin{array}{l}\text { Rockwell Nuclear Materials Source } \\
\text { Data, September } 1978\end{array}$ \\
\hline
\end{tabular}




\begin{tabular}{|c|c|c|c|}
\hline Document Number & Company & Date & Title \\
\hline RHO-CD-78-000521Q & RHO-RL & $01 / 01 / 78$ & $\begin{array}{l}\text { VRA Burial Garden Records Month End } \\
\text { and Source Data January Thru March } \\
1978\end{array}$ \\
\hline RHO-CD-79-000511 & RHO-RL & $01 / 01 / 79$ & $\begin{array}{l}\text { Rockwell Nuclear Materials Source } \\
\text { Data, January } 1979\end{array}$ \\
\hline RHO-CD-79-000511 PT2 & RHO-RL & $01 / 01 / 79$ & $\begin{array}{l}\text { Rockwell Nuclear Materials Source } \\
\text { Data, January } 1979\end{array}$ \\
\hline RHO-CD-79-0005110 & RHO-RL & $10 / 01 / 79$ & $\begin{array}{l}\text { Rockwell Nuclear Materials Source } \\
\text { Data, October } 1979\end{array}$ \\
\hline RHO-CD-79-0005111 & RHO-RL & $12 / 07 / 79$ & $\begin{array}{l}\text { Rockwell Nuclear Materials Source } \\
\text { Data, November } 1979\end{array}$ \\
\hline RHO-CD-79-0005112 & RHO-RL & $12 / 01 / 79$ & $\begin{array}{l}\text { Rockwell Nuclear Materials Source } \\
\text { Data, December } 1979\end{array}$ \\
\hline RHO-CD-79-000512 & RHO-RL & $02 / 01 / 79$ & $\begin{array}{l}\text { Rockwell Nuclear Materials Source } \\
\text { Data, February } 1979\end{array}$ \\
\hline RHO-CD-79-000513. & RHO-RL & $03 / 01 / 79$ & $\begin{array}{l}\text { Rockwell Nuclear Materials Source } \\
\text { Data, March } 1979\end{array}$ \\
\hline RHO-CD-79-000514 & RHO-RL & 04/01/79 & $\begin{array}{l}\text { Rockwell Nuclear Materials Source } \\
\text { Data, April } 1979\end{array}$ \\
\hline RHO-CD-79-000515 & RHO-RL & $05 / 01 / 79$ & $\begin{array}{l}\text { Rockwell Nuclear Materials Source } \\
\text { Data, May } 1979\end{array}$ \\
\hline RHO-CD-79-000516 & RHO-RL & 06/01/79 & $\begin{array}{l}\text { Rockwell Nuclear Materials Source } \\
\text { Data, June } 1979\end{array}$ \\
\hline RHO-CD-79-000517 & RHO-RL & 07/01/79 & $\begin{array}{l}\text { Rockwell Nuclear Materials Source } \\
\text { Data, July } 1979\end{array}$ \\
\hline RHO-CD-79-000518 & RHO-RL & $08 / 01 / 79$ & $\begin{array}{l}\text { Rockwell Nuclear Materials Source } \\
\text { Data, August } 1979\end{array}$ \\
\hline RHO-CD-79-000519 & RHO-RL & $09 / 01 / 79$ & $\begin{array}{l}\text { Rockwell Nuclear Materials Source } \\
\text { Data, September } 1979\end{array}$ \\
\hline RHO-CD-80-0005101 & RHO-RL & $01 / 01 / 80$ & $\begin{array}{l}\text { Rockwell Nuclear Materials Source } \\
\text { Data, January } 1980\end{array}$ \\
\hline
\end{tabular}




\begin{tabular}{|c|c|c|c|}
\hline Document Number & Company & Date & Title \\
\hline RHO-CD-80-0005102 & RHO-RL & $02 / 01 / 80$ & $\begin{array}{l}\text { Rockwell Nuclear Materials Source } \\
\text { Data, February } 1980\end{array}$ \\
\hline RHO-CD-80-0005103 & RHO-RL & $03 / 01 / 80$ & $\begin{array}{l}\text { Rockwell Nuclear Materials Source } \\
\text { Data, March } 1980\end{array}$ \\
\hline RHO-CD-80-0005104 & RHO-RL & $04 / 01 / 80$ & $\begin{array}{l}\text { Rockwell Nuclear Materials Source } \\
\text { Data, April } 1980\end{array}$ \\
\hline RHO-CD-80-0005105 & RHO-RL & $05 / 01 / 80$ & $\begin{array}{l}\text { Rockwell Nuclear Materials Source } \\
\text { Data, May } 1980\end{array}$ \\
\hline RHO-CD-80-0005106 & RHO-RL & $06 / 01 / 80$ & $\begin{array}{l}\text { Rockwell Nuclear Materials Source } \\
\text { Data, June } 1980\end{array}$ \\
\hline RHO-CD-80-0005107 & RHO-RL & $07 / 01 / 80$ & $\begin{array}{l}\text { Rockwell Nuclear Materials Source } \\
\text { Data, July } 1980\end{array}$ \\
\hline RHO-CD-80-0005108 & RHO-RL & $08 / 01 / 80$ & $\begin{array}{l}\text { Rockwell Nuclear Materials Source } \\
\text { Data, August } 1980\end{array}$ \\
\hline RHO-CD-80-0005109 & RHO-RL & $09 / 01 / 80$ & $\begin{array}{l}\text { Rockwell Nuclear Materials Source } \\
\text { Data, September } 1980 \text {. }\end{array}$ \\
\hline RHO-CD-80-0005110 & RHO-RL & $10 / 01 / 80$ & $\begin{array}{l}\text { Rockwell Nuclear Materials Source } \\
\text { Data, October } 1980\end{array}$ \\
\hline RHO-CD-80-00051.11 & RHO-RL & $11 / 01 / 80$ & $\begin{array}{l}\text { Rockwell Nuclear Materials Source } \\
\text { Data, November } 1980\end{array}$ \\
\hline RHO-CD-80-0005112 & RHO-RL & $12 / 01 / 80$ & $\begin{array}{l}\text { Rockwell Nuclear Materials Source } \\
\text { Data, December } 1980\end{array}$ \\
\hline RHO-CD-81-0005101 & RHO-RL & $01 / 01 / 81$ & $\begin{array}{l}\text { Rockwell Nuclear Materials Source } \\
\text { Data, January } 1981\end{array}$ \\
\hline RHO-CD-81-0005102 & RHO-RL & $02 / 01 / 81$ & $\begin{array}{l}\text { Rockwell Nuclear Materials Source } \\
\text { Data, February } 1981\end{array}$ \\
\hline RHO-CD-81-0005103 & RHO-RL & $03 / 01 / 81$ & $\begin{array}{l}\text { Rockwell Nuclear Materials Source } \\
\text { Data, March } 1981\end{array}$ \\
\hline RHO-CD-81-0005104 & RHO-RL & $04 / 01 / 81$ & $\begin{array}{l}\text { Rockwell Nuclear Materials Source } \\
\text { Data, April } 1981\end{array}$ \\
\hline
\end{tabular}




\begin{tabular}{|c|c|c|c|}
\hline Document Number & Company & Date & Title \\
\hline RHO-CD-81-0005105 & RHO-RL & 05/01/81 & $\begin{array}{l}\text { Rockwell Nuclear Materials Source } \\
\text { Data, May } 1981\end{array}$ \\
\hline RHO-CD-81-0005106 & RHO-RL & $06 / 01 / 81$ & $\begin{array}{l}\text { Rockwell Nuclear Materials Source } \\
\text { Data, June } 1981^{\circ}\end{array}$ \\
\hline RHO-CD-81-0005107 & RHO-RL & $07 / 01 / 81$ & $\begin{array}{l}\text { Rockwell Nuclear Materials Source } \\
\text { Data, July } 1981\end{array}$ \\
\hline RHO-CD-81-0005108 & RHO-RL & $08 / 01 / 81$ & $\begin{array}{l}\text { Rockwell Nuclear Materials Source } \\
\text { Data, August } 1981\end{array}$ \\
\hline RHO-CD-81-0005109 & RHO-RL & 09/01/81 & $\begin{array}{l}\text { Rockwell Nuclear Materials Source } \\
\text { Data, September } 1981\end{array}$ \\
\hline RHO-CP-EV-0001ORD & & 07/18/85 & FY 1986 Budget Impact Analysis \\
\hline RHO-CP-EV-00014 & & $04 / 23 / 86$ & $\begin{array}{l}\text { Gramm Rudman Hollings Impacts to } \\
\text { FY } 87 \text { and FY } 88 \text { Materials Production } \\
\text { Program Classified Attachment to } \\
\text { Lt \#30409 R1 }\end{array}$ \\
\hline RHO-CP-PL-00046 & RHO-RL & $12 / 12 / 86$ & $\begin{array}{l}\text { Chemical Processing FY } 1987 \\
\text { Production Commitments and Forecasts }\end{array}$ \\
\hline RHO-CP-PL-85-00043 & & $11 / 06 / 85$ & $\begin{array}{l}\text { Chemical Processing Program } \\
\text { Review - October 31, } 1985 \text { FY } 1986 \\
\text { Production Data }\end{array}$ \\
\hline RHO-CP-SR-00003 & & 04/01/82 & $\begin{array}{l}\text { FY } 1982 \text { Midyear Review Chemical } \\
\text { Processing Program }\end{array}$ \\
\hline RHO-CP-SR-00012 & & $05 / 15 / 85$ & $\begin{array}{l}\text { Chemical Processing Program Extended } \\
\text { Life Cost Benefits Analysis }\end{array}$ \\
\hline RHO-CP-SR-00013 & e & $05 / 31 / 85$ & $\begin{array}{l}\text { Fiscal Year } 1986 \text { Budget Impact } \\
\text { Analysis }\end{array}$ \\
\hline RHO-FI-EV-00010 & RHO-RL & $02 / 26 / 87$ & $\begin{array}{l}\text { Information Resources Control Program } \\
\text { Damage Assessment } 87-2 \text { Classified } \\
\text { Document Inventory and Initial } \\
\text { Assessment }\end{array}$ \\
\hline RHO-PO-NB-00005 & & $12 / 17 / 84$ & Nuclear Material Source Documentation \\
\hline
\end{tabular}




\begin{tabular}{|c|c|c|c|}
\hline Document Number & Company & Date & Title \\
\hline RHO-PP-VS-00001 & RHO-RL & $03 / 22 / 84$ & $\begin{array}{l}\text { Onsite Shipment of Plutonium Oxide } \\
\text { Sample Without Nuclear Materials } \\
\text { Release Unusual Occurrence Report } \\
\text { No. } 84-18\end{array}$ \\
\hline RHO-PP-VS-00001 REV1 & RHO-RL & $04 / 24 / 84$ & $\begin{array}{l}\text { Onsite Shipment of Plutonium Oxide } \\
\text { Sample Without Nuclear Materials } \\
\text { Release Unusual Occurrence Report } \\
\text { \#84-18 } \\
\end{array}$ \\
\hline RHO-QA-PL-00016 & RHO-RL & $12 / 07 / 84$ & $\begin{array}{l}\text { Plutonium-Uranium Analysis on } \\
\text { Samples from Selected UNC Fuel } \\
\text { Elements }\end{array}$ \\
\hline RHO-QA-PL-00016 & UNC & $12 / 07 / 84$ & $\begin{array}{l}\text { Plutonium-Uranium Analysis on } \\
\text { Samples from Selected UNC Nuclear } \\
\text { Industries Fuel Elements }\end{array}$ \\
\hline RHO-RE-EV-00007 & RHO-RL & $03 / 01 / 82$ & $\begin{array}{l}\text { Chemical Separation and Waste } \\
\text { Management for Hanford Augmented } \\
\text { Material Production Alternatives }\end{array}$ \\
\hline RHO-RE-NB-00124WP & RHO-RL & $12 / 06 / 83$ & $\begin{array}{l}\text { Electrochemical Dissolution of Pu Metal } \\
\text { Extraction }\end{array}$ \\
\hline RHO-RE-NB-00168 & RHO-RL & $\begin{array}{c}12 / 07 / 84 \\
.\end{array}$ & FY-85 PRF Sample Log \\
\hline RHO-RE-SR-00018PT1 +2 & & $02 / 24 / 83$ & TRAC Fuels Source Data (TAPES) \\
\hline RHO-SI-EV-00001 & RHO-RL & 03/04/85 & $\begin{array}{l}\text { SIS Chemical Processing Material } \\
\text { Balance Flowsheet }\end{array}$ \\
\hline RHO-SI-FL-00001 & RHO-RL & $06 / 07 / 85$ & $\begin{array}{l}\text { Purex SIS Chemical Material Balance } \\
\text { Flowsheet Nitrate Feed - Metal Line }\end{array}$ \\
\hline RHO-SI-FL-00002 & RHO-RL & $06 / 07 / 85$ & $\begin{array}{l}\text { Purex SIS Chemical Material Balance } \\
\text { Flowsheet Oxide Feed - Metal Line }\end{array}$ \\
\hline RHO-SI-FL-00003 & RHO-RL & $06 / 07 / 85$ & $\begin{array}{l}\text { Purex SIS Chemical Material Balance } \\
\text { Flowsheet Balance of Plant }\end{array}$ \\
\hline RHO-SS-EV-00045 & RHO-RL & $07 / 23 / 84$ & $\begin{array}{l}\text { Suggestion Number } 97137, \text { A.C. } \\
\text { Morganthaler }\end{array}$ \\
\hline
\end{tabular}




\begin{tabular}{|c|c|c|c|}
\hline Document Number & Company & Date & Title \\
\hline RHO-SS-MA-00046 & RHO-RL & $01 / 01 / 85$ & $\begin{array}{l}\text { Installation of a Chemical Smoke Access } \\
\text { Denial System in Building 2736-ZB, } \\
200 \text { West Area }\end{array}$ \\
\hline $\begin{array}{l}\text { RHO-SS-MA-00046 } \\
\text { W/O ENC }\end{array}$ & RHO-RL & 01/01/85 & $\begin{array}{l}\text { Installation of a Chemical Smoke Access } \\
\text { Denial System in Building 2736-ZB, } \\
200 \text { West Area }\end{array}$ \\
\hline $\begin{array}{l}\text { RHO-SS-MA-00046 } \\
\text { W/O ENC }\end{array}$ & RHO-RL & 01/01/85 & $\begin{array}{l}\text { Installation of a Chemical Smoke Access } \\
\text { Denial System in Building 2736-ZB, } \\
200 \text { West Area }\end{array}$ \\
\hline $\begin{array}{l}\text { RHO-SS-PL-00007 } \\
\text { REV2 ADD }\end{array}$ & WHC-RL & 07/01/88 & $\begin{array}{l}\text { Chemical Storage, Aqueous Make-Up } \\
\text { and Chemical Distribution Sabotage } \\
\text { Recovery Plan }\end{array}$ \\
\hline RHO-SS-PL-00098 & RHO-RL & $07 / 25 / 85$ & $\begin{array}{l}\text { Assessment of Impacts for Hanford } \\
\text { Patrol Budget Elements }\end{array}$ \\
\hline RHO-SS-PL-00123 & RHO-RL & 09/01/86 & $\begin{array}{l}\text { Technical Descriptions Estimated Costs } \\
\text { and Impacts of Purex Facility N Cell } \\
\text { Safeguards Upgrades Reports }\end{array}$ \\
\hline RHO-SS-PL-00124 & RHO-RL & $09 / 01 / 86$ & $\begin{array}{l}\text { Technical Descriptions Estimated Costs } \\
\text { and Impacts of Plutonium Finishing } \\
\text { Plant Remote Mechanical "C". Line } \\
\text { SFGDS Upgrades Reports }\end{array}$ \\
\hline RHO-SS-SD-00004 & & $08 / 28 / 86$ & RHO NM Source Data Working Papers \\
\hline RHO-SS-SD-81-0005110 & RHO-RL & 10/01/81. & $\begin{array}{l}\text { Rockwell Nuclear Materials Source } \\
\text { Data, October } 1981\end{array}$ \\
\hline RHO-SS-SD-81-0005111 & & $11 / 01 / 81$ & $\begin{array}{l}\text { Rockwell Nuclear Materials Source } \\
\text { Data, November } 1981\end{array}$ \\
\hline RHO-SS-SD-81-0005112 & RHO-RL & $12 / 01 / 81$ & $\begin{array}{l}\text { Rockwell Nuclear Materials Source } \\
\text { Data, December } 1981\end{array}$ \\
\hline RHO-SS-SD-82-0005101 & RHO-RL & $01 / 31 / 82$ & $\begin{array}{l}\text { Rockwell Nuclear Materials Source. } \\
\text { Data, January } 1982\end{array}$ \\
\hline RHO-SS-SD-82-0005:102 & RHO-RL & $02 / 01 / 82$ & $\begin{array}{l}\text { Rockwell Nuclear Materials Source } \\
\text { Data, February } 1982\end{array}$ \\
\hline
\end{tabular}


Document Number

RHO-SS-SD-82-0005103

RHO-SS-SD-82-0005104

RHO-SS-SD-82-0005105

RHO-SS-SD-82-0005106

RHO-SS-SD-82-0005107

RHO-SS-SD-82-0005108

RHO-SS-SD-82-0005109

RHO-SS-SD-82-0005110

RHO-SS-SD-82-0005111

RHO-SS-SD-82-0005112

RHO-SS-SD-83-0005101

RHO-SS-SD-83-0005102

RHO-SS-SD-83-0005103

RHO-SS-SD-83-0005104

RHO-SST-SD-83-0005106[5?]
Company

RHO-RL

RHO-RL

$04 / 01 / 82$

RHO-RL

RHO-RL

RHO-RL

RHO-RL

RHO-RL

RHO-RL

RHO-RL

RHO-RL

$12 / 01 / 82$

RHO-RL 01/01/83

RHO-RL

$02 / 01 / 83$

RHO-RL

$04 / 12 / 82$

RHO-RL

$05 / 16 / 82$

RHO-RL

Date

$03 / 01 / 82$

$05 / 01 / 82$

$06 / 01 / 82$

$07 / 01 / 82$

$08 / 01 / 82$

$09 / 01 / 82$

$10 / 01 / 82$

$11 / 01 / 82$ Data, October 1982

Rockwell Nuclear
Data, August 1982

Rockwell Nuclear Materials Source Data, July 1982

Rockwell Nuclea
Data, May 1982

Rockwell Nuclear Materials Source Data, June 1982

Rockwell Nuclear Materials Source Data, September 1982

Rockwell Nuclear Materials Source

Rockwell Nuclear Materials Source Data, November 1982

Rockwell Nuclear Materials Source Data, December 1982

Rockwell Nuclear Materials Source Data, January 1983

Rockwell Nuclear Materials Source Data, February 1983

Rockwell Nuclear Materials Source Data, March 1983

Rockwell Nuclear Materials Source Data, April 1983

05/01/83 Rockwell Nuclear Materials Source Data, May 1983 


\begin{tabular}{|c|c|c|c|}
\hline Document Number & Company & Date & Title \\
\hline RHO-SS-SD-83-0005106 & RHO-RL & $06 / 01 / 83$ & $\begin{array}{l}\text { Rockwell Nuclear Materials Source } \\
\text { Data, June } 1983\end{array}$ \\
\hline RHO-SS-SD-83-0005107 & RHO-RL & $07 / 01 / 83$ & $\begin{array}{l}\text { Rockwell Nuclear Materials Source } \\
\text { Data, July } 1983\end{array}$ \\
\hline RHO-SS-SD-83-0005108 & RHO-RL & $08 / 01 / 83$ & $\begin{array}{l}\text { Rockwell Nuclear Materials Source } \\
\text { Data, August } 1983\end{array}$ \\
\hline RHO-SS-SD-83-0005109 & RHO-RL & $09 / 01 / 83$ & $\begin{array}{l}\text { Rockwell Nuclear Materials Source } \\
\text { Data, September } 1983\end{array}$ \\
\hline RHO-SS-SD-83-0005110 & RHO-RL & $10 / 01 / 83$ & $\begin{array}{l}\text { Rockwell Nuclear Materials Source } \\
\text { Data, October } 1983\end{array}$ \\
\hline RHO-SS-SD-83-0005111 & RHO-RL & $11 / 01 / 83$ & $\begin{array}{l}\text { Rockwell Nuclear Materials Source } \\
\text { Data, November } 1983\end{array}$ \\
\hline RHO-SS-SD-83-0005112 & RHO-RL & $12 / 01 / 83$ & $\begin{array}{l}\text { Rockwell Nuclear Materials Source } \\
\text { Data, December } 1983\end{array}$ \\
\hline RHO-SS-SD-84-0005101 & RHO-RL & $01 / 19 / 84$ & $\begin{array}{l}\text { Rockwell Nuclear Materials Source } \\
\text { Data, January } 1984\end{array}$ \\
\hline RHO-SS-SD-84-0005102 & RHO-RL & $02 / 01 / 84$ & $\begin{array}{l}\text { Rockwell Nuclear Materials Source } \\
\text { Data, February } 1984\end{array}$ \\
\hline RHO-SS-SD-84-0005103 & RHO-RL & $03 / 01 / 84$ & $\begin{array}{l}\text { Rockwell Nuclear Materials Source } \\
\text { Data, March } 1984\end{array}$ \\
\hline RHO-SS-SD-84-0005104 & RHO-RL & $04 / 01 / 84$ & $\begin{array}{l}\text { Rockwell Nuclear Materials Source } \\
\text { Data, April } 1984\end{array}$ \\
\hline RHO-SS-SD-84-0005105 & RHO-RL & $05 / 01 / 84$ & $\begin{array}{l}\text { Rockwell Nuclear Materials Source } \\
\text { Data, May } 1984\end{array}$ \\
\hline RHO-SS-SD-84-0005106 & RHO-RL & $06 / 01 / 84$ & $\begin{array}{l}\text { Rockwell Nuclear Materials Source } \\
\text { Data, June } 1984\end{array}$ \\
\hline RHO-SS-SD-84-0005107 & RHO-RL & $07 / 01 / 84$ & $\begin{array}{l}\text { Rockwell Nuclear Materials Source } \\
\text { Data, July } 1984\end{array}$ \\
\hline RHO-SS-SD-84-0005108 & RHO-RL & $08 / 01 / 84$ & $\begin{array}{l}\text { Rockwell Nuclear Materials Source } \\
\text { Data, August } 1984\end{array}$ \\
\hline & & 21 & • \\
\hline
\end{tabular}


Document Number

RHO-SS-SD-84-0005109

RHO-SS-SD-84-0005110

RHO-SS-SD-84-0005111

RHO-SS-SD-84-0005112

RHO-SS-SD-85-0005101

RHO-SS-SD-85-0005102

RHO-SS-SD-85-0005103

RHO-SS-SD-85-0005104

RHO-SS-SD-85-0005105

RHO-SS-SD-85-0005106

RHO-SS-SD-85-0005107

RHO-SS-SD-85-0005108

RHO-SS-SD-85-0005109

RHO-SS-SD-85-0005110

RHO-SS-SD-85-0005111
Company

RHO-RL

RHO-RL

RHO-RL

RHO-RL

RHO-RL

RHO-RL

RHO-RL

RHO-RL

RHO-RL

RHO-RL $\quad 06 / 01 / 85$

RHO-RL

RHO-RL

09/05/85

RHO-RL

$09 / 30 / 85$

RHO-RL

$11 / 05 / 85$

RHO-RL . 11/01/85
Title

Rockwell Nuclear Materials Source

Data, September 1984

Rockwell Nuclear Materials Source Data, October 1984

Rockwell Nuclear Materials Source Data, November 1984

Rockwell Nuclear Materials Source Data, December 1984

Rockwell Nuclear Materials Source Data, January 1985

Rockwell Nuclear Materials Source Data, February 1985

Rockwell Nuclear Materials Source Data, March 1985

Rockwell Nuclear Materials Source Data, April 1985

Rockwell Nuclear Materials Source Data, May 1985

Rockwell Nuclear Materials Source Data, June 1985

Rockwell Nuclear Materials Source Data, July 1985

Rockwell Nuclear Materials Source Data, August 1985

Rockwell Nuclear Materials Source Data, September 1985

Rockwell Nuclear Materials Source Data, October 1985

Rockwell Nuclear Materials Source Data, November 1985 


\begin{tabular}{|c|c|c|c|}
\hline Document Number & Company & Date & Title \\
\hline RHO-SS-SD-85-0005112 & RHO-RL & $12 / 01 / 85$ & $\begin{array}{l}\text { Rockwell Nuclear Materials Source } \\
\text { Data, December } 1985\end{array}$ \\
\hline RHO-SS-SD-86-0005101 & RHO-RL & $01 / 01 / 86$ & $\begin{array}{l}\text { Rockwell Nuclear Materials Source } \\
\text { Data, January } 1986\end{array}$ \\
\hline RHO-SS-SD-86-0005102 & RHO-RL & $02 / 01 / 86$ & $\begin{array}{l}\text { Rockwell Nuclear Materials Source } \\
\text { Data, February } 1986\end{array}$ \\
\hline RHO-SS-SD-86-0005103 & RHO-RL & $03 / 01 / 86$ & $\begin{array}{l}\text { Rockwell Nuclear Materials Source } \\
\text { Data, March } 1986\end{array}$ \\
\hline RHO-SS-SD-86-0005104 & RHO-RL & $04 / 01 / 86$ & $\begin{array}{l}\text { Rockwell Nuclear Materials Source } \\
\text { Data, April } 1986\end{array}$ \\
\hline RHO-SS-SD-86-0005105 & RHO-RL & $06 / 12 / 86$ & $\begin{array}{l}\text { Rockwell Nuclear Materials Source } \\
\text { Data, May } 1986\end{array}$ \\
\hline RHO-SS-SD-86-0005106 & RHO-RL & $06 / 01 / 86$ & $\begin{array}{l}\text { Rockwell Nuclear Materials Source } \\
\text { Data, June } 1986\end{array}$ \\
\hline RHO-SS-SD-86-0005107 & RHO-RL & $07 / 01 / 86$ & $\begin{array}{l}\text { Rockwell Nuclear Materials Source } \\
\text { Data, July } 1986\end{array}$ \\
\hline RHO-SS-SD-86-0005108 & RHO-RL & $08 / 01 / 86$ & $\begin{array}{l}\text { Rockwell Nuclear Materials Source } \\
\text { Data, August } 1986\end{array}$ \\
\hline RHO-SS-SD-86-0005109 & RHO-RL & $09 / 01 / 86$ & $\begin{array}{l}\text { Rockwell Nuclear Materials Source } \\
\text { Data, September } 1986\end{array}$ \\
\hline RHO-SS-SD-86-0005110 & RHO-RL & $10 / 01 / 86$ & $\begin{array}{l}\text { Rockwell Nuclear Materials Source } \\
\text { Data, October } 1986\end{array}$ \\
\hline RHO-SS-SD-86-0005111 & RHO-RL & $11 / 01 / 86$ & $\begin{array}{l}\text { Rockwell Nuclear Materials Source } \\
\text { Data, November } 1986\end{array}$ \\
\hline RHO-SS-SD-86-0005112 & RHO-RL & $12 / 01 / 86$ & $\begin{array}{l}\text { Rockwell Nuclear Materials Source } \\
\text { Data, December } 1986\end{array}$ \\
\hline RHO-SS-SD-87-0005101 & RHO-RL & $01 / 01 / 87$ & $\begin{array}{l}\text { Rockwell Nuclear Materials Source } \\
\text { Data, January } 1987\end{array}$ \\
\hline RHO-SS-SD-87-0005102 & RHO-RL & $02 / 01 / 87$ & $\begin{array}{l}\text { Rockwell Nuclear Materials Source } \\
\text { Data, February } 1987\end{array}$ \\
\hline
\end{tabular}




\begin{tabular}{|c|c|c|c|}
\hline Document Number & Company & Date & Title \\
\hline RHO-SS-SD-87-0005103 & RHO-RL & 03/01/87 & $\begin{array}{l}\text { Rockwell Nuclear Materials Source } \\
\text { Data, March } 1987\end{array}$ \\
\hline RHO-SS-SD-87-0005104 & RHO-RL & $04 / 01 / 87$ & $\begin{array}{l}\text { Rockwell Nuclear Materials Source } \\
\text { Data, April } 1987\end{array}$ \\
\hline RHO-SS-SD-87-0005105 & RHO-RL & $05 / 01 / 87$ & $\begin{array}{l}\text { Rockwell Nuclear Materials Source } \\
\text { Data, May } 1987\end{array}$ \\
\hline RHO-SS-SD-87-0005106 & RHO-RL & $06 / 01 / 87$ & $\begin{array}{l}\text { Rockwell Nuclear Materials Source } \\
\text { Data, June } 1987\end{array}$ \\
\hline RHO-SS-SD-87-0005107 & & $07 / 01 / 87$ & $\begin{array}{l}\text { Rockwell Nuclear Materials Source } \\
\text { Data, July } 1987^{\prime}\end{array}$ \\
\hline RL-DPO-87-00018MAY & DOE-RL & $06 / 23 / 87$ & $\begin{array}{l}\text { RL Monthly Production Report UNC } \\
\text { Nuclear Industries - N Reactor Rockwell } \\
\text { Hanford Operations - Chemical } \\
\text { Processing }\end{array}$ \\
\hline RL-HQ-00104 & DOE-HQ & $06 / 30 / 86$ & $\begin{array}{l}\text { Generation and Handling of Mixtures of } \\
\text { Chemical and Radioactive Wastes by } \\
\text { Naval Reactors }\end{array}$ \\
\hline RL-HQ-00403 & DOE-HQ & $05 / 03 / 89$ & $\begin{array}{l}\text { Annual Compilation of Security } \\
\text { Evaluations (OSE) Findings for the } \\
\text { Period of } 9 / 87 \text { Through } 2 / 89\end{array}$ \\
\hline RLO-HQ-00018 & DOE-HQ & $12 / 18 / 85$ & Material Procured from Foreign Sources \\
\hline SAB20001641B003 & DOE-HQ & $04 / 01 / 92$ & $\begin{array}{l}\text { Classification of Radioactive and } \\
\text { Hazardous Materials in the Nuclear } \\
\text { Weapon Compels - Part } 2 \text { - Appendices } \\
\text { (U) }\end{array}$ \\
\hline SGS-C-00588 & DOE-RL & $06 / 20 / 79$ & $\begin{array}{l}\text { Control Over Movement of Radioactive } \\
\text { Material }\end{array}$ \\
\hline SK-2-61241 SH1 RO A & RHO & $09 / 25 / 84$ & $\begin{array}{l}\text { Chemical Processing Block Flow } \\
\text { Diagram Base Case (Aperture Card) }\end{array}$ \\
\hline SK-2-61241 SH1 RO M & RHO & $04 / 22 / 85$ & $\begin{array}{l}\text { Chemical Processing Block Flow } \\
\text { Diagram Base Case }\end{array}$ \\
\hline
\end{tabular}




\begin{tabular}{|c|c|c|c|}
\hline Document Number & Company & Date & Title \\
\hline SK-2-61241 SH2 RO A & RHO & $08 / 27 / 84$ & $\begin{array}{l}\text { Chemical Processing Block Flow } \\
\text { Diagram Base Case (Aperture Card) }\end{array}$ \\
\hline SK-2-61241 SH2 RO M & RHO & $04 / 22 / 85$ & $\begin{array}{l}\text { Chemical Processing Block Flow } \\
\text { Diagram Base Case }\end{array}$ \\
\hline SK-2-61241 SH3 RO A & RHO & $08 / 27 / 84$ & $\begin{array}{l}\text { Chemical Processing Block Flow } \\
\text { Diagram Base Case (Aperture Card) }\end{array}$ \\
\hline SK-2-61241 SH3 RO M & RHO & $04 / 22 / 85$ & $\begin{array}{l}\text { Chemical Processing Block Flow } \\
\text { Diagram Base Case }\end{array}$ \\
\hline SK-2-61242 SH1 RO A & RHO & $04 / 19 / 85$ & $\begin{array}{l}\text { Chemical Processing Block Flow } \\
\text { Diagram Alternate Case (Aperture Card) }\end{array}$ \\
\hline SK-2-61242 SH1 RO M & RHO & $04 / 22 / 85$ & $\begin{array}{l}\text { Chemical Processing Block Flow } \\
\text { Diagram Alternate Case }\end{array}$ \\
\hline SK-2-61242 SH2 RO A & RHO & $04 / 19 / 85$ & $\begin{array}{l}\text { Chemical Processing Block Flow } \\
\text { Diagram Alternate Case (Aperture Card) }\end{array}$ \\
\hline SK-2-61242 SH2 RO M & RHO & $04 / 22 / 85$ & $\begin{array}{l}\text { Chemical Processing Block Flow } \\
\text { Diagram Alternate Case }\end{array}$ \\
\hline SK-2-61242 SH3 RO A & RHO & $04 / 19 / 85$ & $\begin{array}{l}\text { Chemical Processing Block Flow } \\
\text { Diagram Alternate Case (Aperture Card) }\end{array}$ \\
\hline SK-2-61242 SH3 RO M & RHO & $04 / 22 / 85$ & $\begin{array}{l}\text { Chemical Processing Block Flow } \\
\text { Diagram Alternate Case }\end{array}$ \\
\hline SK-2-61615 SH1 RO A & RHO & 09/03/86 & $\begin{array}{l}\text { Purex/SIS Chemical Material Balance } \\
\text { Block Flow Diagram (Aperture Card) }\end{array}$ \\
\hline SK-2-61615 SH1 RO M & RHO & 09/05/86 & $\begin{array}{l}\text { Purex/SIS Chemical Material Balance } \\
\text { Block Flow Diagram }\end{array}$ \\
\hline SK-2-61615 SH2 RO A & RHO & 09/03/86 & $\begin{array}{l}\text { Purex/SIS Chemical Material Balance } \\
\text { Block Flow Diagram (Aperture Card) }\end{array}$ \\
\hline SK-2-61615 SH2 RO M & RHO & 09/05/86 & $\begin{array}{l}\text { Purex/SIS Chemical Material Balance } \\
\text { Block Flow Diagram }\end{array}$ \\
\hline SḲ-2-61615 SH3 RO A & RHO & 09/03/86 & $\begin{array}{l}\text { Purex/SIS Chemical Material Balance } \\
\text { Block Flow Diagram (Aperture Card) }\end{array}$ \\
\hline
\end{tabular}


Document Number

SK-2-61615 SH3 RO M

SK-2-61621 SH1 RO A

SK-2-61621 SH1 RO M

SK-2-61621 SH2 RO A

SK-2-61621 SH2 RO M

SK-2-61621 SH3 RO A

SK-2-61621 SH3 RO M

SK-2-61621 SH4 RO A

SK-2-61621 SH4 RO M

SK-2-61621 SH5 RO A

SK-2-61621 SH5 RO M

SK-2-61637 SH1 RO A

SK-2-61637 SH1 RO M

SK-2-61637 SH2 RO A

SK-2-61637 SH2 RO M

Company $\frac{\text { Date }}{09 / 05 / 86}$

RHO

RHO

RHO

RHO

RHO

RHO

RHO

RHO

RHO

RHO

RHO

RHO

RHO

RHO

07/01/85

$07 / 10 / 85$

07/01/85

07/10/85

07/01/85

$07 / 10 / 85$

07/01/85

$07 / 10 / 85$

07/01/85

$07 / 10 / 85$

09/01/86

$01 / 21 / 85$

09/01/86

$02 / 14 / 85$
SIS Chemical Processing Material Balance Flowsheet (Aperture Card)

Purex/SIS Chemical Material Balance Block Flow Diagram

SIS Chemical Processing Flowsheet 200 West Pyro (Aperture Card)

SIS Chemical Processing Flowsheet 200 West Pyro

SIS Chemical Processing Flowsheet 200 West Pyro (Aperture Card)

SIS Chemical Processing Flowsheet 200 West Pyro

SIS Chemical Processing Flowsheet 200 West Pyro (Aperture Card)

SIS Chemical Processing Flowsheet 200 West Pyro

SIS Chemical Processing Flowsheet 200 West Pyro (Aperture Card)

SIS Chemical Processing Flowsheet 200 West Pyro

SIS Chemical Processing Flowsheet 200 West Pyro (Aperture Card)

SIS Chemical Processing Flowsheet 200 West Pyro

SIS Chemical Processing Material Balance Flowsheet (Aperture Card)

SIS Chemical Processing Material Balance Flowsheet

SIS Chemical Processing Material Balance Flowsheet 


\begin{tabular}{|c|c|c|c|}
\hline Document Number & Company & Date & Title \\
\hline SK-2-61637 SH3 RO A & RHO & 09/01/86 & $\begin{array}{l}\text { SIS Chemical Processing Material } \\
\text { Balance Flowsheet (Aperture Card) }\end{array}$ \\
\hline SK-2:61637 SH3 RO M & RHO & $02 / 14 / 85$ & $\begin{array}{l}\text { SIS Chemical Processing Material } \\
\text { Balance Flowsheet }\end{array}$ \\
\hline SR-BP-81-00042 WD & & $08 / 06 / 81$ & $\begin{array}{l}\text { Impact of Weapons Grade Plutonium } \\
\text { Specifications Upon Sr Plant Production } \\
\text { Schedule }\end{array}$ \\
\hline TT-01045 & DOE-RL & $05 / 08 / 79$ & $\begin{array}{l}\text { Investigation of Neutron Source } \\
\text { Received by HEDL from Johnston } \\
\text { Island }\end{array}$ \\
\hline UNI-I-00368VOL1 & & $01 / 20 / 81$ & $\begin{array}{l}\text { Compilation of Mass Flow Data for the } \\
\text { RPR Project }\end{array}$ \\
\hline UNI-I-00368VOL2 & - & $01 / 20 / 81$ & $\begin{array}{l}\text { Compilation of Mass Flow Data for the } \\
\text { RPR Project }\end{array}$ \\
\hline UNI-I-00368VOL3 & & $01 / 20 / 81$ & $\begin{array}{l}\text { Compilation of Mass Flow Data for the } \\
\text { RPR Project }\end{array}$ \\
\hline UNI-N-00368VOL1 & UNC-RL & $01 / 20 / 81$ & $\begin{array}{l}\text { Compilation of Mass Flow Data for the } \\
\text { RPR Project }\end{array}$ \\
\hline UNI-Ṇ-00368VOL2 & UNC-RL & $01 / 20 / 81$ & $\begin{array}{l}\text { Compilation of Mass Flow Data for the } \\
\text { RPR Project }\end{array}$ \\
\hline UNI-N-00368VOL3 & UNC-RL & $01 / 20 / 81$ & $\begin{array}{l}\text { Compilation of Mass Flow Data for the } \\
\text { RPR Project }\end{array}$ \\
\hline UNI-01030 & UNC-RL & 04/01/78 & $\begin{array}{l}\text { Nuclear Materials Inventory and Source } \\
\text { Data, April Thru June } 1978\end{array}$ \\
\hline UNI-01120 & UNC-RL & $07 / 01 / 78$ & $\begin{array}{l}\text { Nuclear Materials Inventory and Source } \\
\text { Data, July Thru September } 1978\end{array}$ \\
\hline UNI-01156 & UNC-RL & $10 / 01 / 78$ & $\begin{array}{l}\text { Nuclear Materials Inventory and Source } \\
\text { Data, October Thru December } 1978\end{array}$ \\
\hline UNI-01225 & UNC-RL & $01 / 01 / 79$ & $\begin{array}{l}\text { Nuclear Materials Inventory and Source } \\
\text { Data, January Thru March } 1979\end{array}$ \\
\hline
\end{tabular}


Document Number

UNI-01304

UNI-01542 18

UNI-01542 19

UNI-0154222

UṆI-0154223

UNI-0154224

UNI-0154225

UNI-0154226

UNI-0154227

UNI-0154228

UNI-0154229

UNI-0154230

UNI-0154231

UNI-0154232

UNI-0154233 $\frac{\text { Company }}{\text { UNC-RL }} \frac{\text { Date }}{04 / 01 / 79}$

UNC-RL $\quad 07 / 31 / 83$

UNC-RL $\quad 08 / 31 / 83$

UNC-RL

$11 / 30 / 83$

UNC-RL

$12 / 31 / 83$

UNC-RL $\quad 01 / 31 / 84$

UNC-RL

$02 / 29 / 84$

UNC-RL

$03 / 31 / 84$

UNC-RL

$04 / 30 / 84$

UNC-RL

$05 / 31 / 84$

UNC-RL

$06 / 30 / 84$

UNC-RL

$07 / 31 / 84$

UNC-RL

$08 / 31 / 84$

UNC-RL

$09 / 30 / 84$

UNC-RL $\quad \cdot 11 / 12 / 84$
Title

Nuclear Material Inventory and Source Data, April, May, June 1979

Nuclear Materials Inventory and Source Data, July 1983

Nuclear Materials Inventory and source Data, August 1983

Nuclear Materials Inventory and Source Data, November 1983

Nuclear Materials Inventory and Source Data, December 1983

Nuclear Materials Inventory and Source Data, January 1984

Nuclear Materials Inventory and Source Data, February 1984

Nuclear Materials Inventory and Source Data, March 1984

Nuclear Materials Inventory and Source Data, April 1984 .

Nuclear Materials Inventory and Source Data, May 1984

Nuclear Materials Inventory and Source Data, June 1984

Nuclear Materials Inventory and Source Data, July 1984

Nuclear Materials Inventory and Source Data, August 1984

Nuclear Materials Inventory and Source Data, September 1984

Nuclear Materials Inventory and Source Data, October 1984 
Document Number

UNI-0154236
UNI-0154237
UNI-0154238

UNI-0154239

UNI-0154240

UNI-0154241

UNI-0154242

UNI-0154243

UNI-0154244

UNI-0154245

UNI-0154246

UNI-0154 247

UNI-0154248

UNI-0154249

UNI-0154250
Company

Date

UNC-RL

$01 / 31 / 84$

UNC-RL

$02 / 28 / 85$

UNC-RL

$03 / 31 / 85$

UNC-RL

$04 / 30 / 85$

UNC-RL

$05 / 31 / 85$

UNC-RL

UNC-RL

UNC-RL

UNC-RL

UNC-RL

UNC-RL

UNC-RL

$12 / 31 / 85$

$08 / 31 / 85$

$06 / 30 / 85$

$07 / 31 / 85$

$08 / 31 / 85$

$08 / 31 / 85$

$10 / 31 / 85$

$11 / 30 / 85$

Monthly Nuclear Materials Inventory and Source Data, November 1985

Materials Inventory and Source Data

Monthly Nuclear Materials Inventory and Source Data, October 1983

June 1985 Monthly Nuclea
Inventory and Source Data

July 1985 Monthly Nuclear Materials Inventory and Source Data

August 1985 Monthly Nuclear Materials Inventory and Source Data

January 1985 Monthly Nuclear

Materials Inventory and Source Data

February 1985 Monthly Nuclear Materials Inventory and Source Data

March 1985 Monthly Nuclear Materials Inventory and Source Data

April 1985 Monthly Nuclear Materials Inventory and Source Data

May 1985 Monthly Nuclear Materials Inventory and Source Data .

Monthly Nuclear Materials Inventory

Monthly Nuclear Materials Inventory and Source Data, December 1985

UNC-RL $\quad 01 / 13 / 86$

Monthly Nuclear Materials Inventory and Source Data, January 1986
UNC-RL 02/28/86 Monthly Nuclear Materials Inventory and Source Data, February 1986

UNC-RL 03/31/86

Monthly Nuclear Materials Inventory and Source Data, March 1986 
Document Number

UNI-0154251

UNI-0154252

UNI-0154253

UNI-0154254

UNI-0154255

UNI-0154256

UNI-0154257

UNI-0154258

UNI-0154259

UNI-0154260

UNI-0154261

UNI-0154262

UNI-0154263

UNI-0154264

UNI-0154265

$\frac{\text { Company }}{\text { UNC-RL }} \quad \frac{\text { Date }}{04 / 30 / 86}$

UNC-RL

$05 / 31 / 86$

UNC-RL

$06 / 30 / 86$

UNC-RL

$07 / 31 / 86$

UNC-RL

$08 / 30 / 86$

UNC-RL

$09 / 30 / 86$

UNC-RL

$10 / 31 / 86$

UNC-RL

$11 / 30 / 86$

UNC-RL

$12 / 31 / 86$

UNC-RL

$01 / 31 / 87$

UNC-RL $\quad 02 / 28 / 87$

UNC-RL

$03 / 31 / 87$

UNC-RL

04/30/87

UNC-RL

$05 / 31 / 87$

UNC-RL
Title

Monthly Nuclear Materials Inventory and Source Data, April 1986

Monthly Nüclear Materials Inventory and Source Data, May 1986

Monthly Nuclear Materials Inventory and Source Data, June 1986

Monthly Nuclear Materials Inventory and Source Data; July 1986

Monthly Nuclear Materials Inventory and Source Data, August 1986

Monthly Nuclear Materials Inventory and Source Data, September 1986

Monthly Nuclear Materials Inventory and Source Data, October 1986

Monthly Nuclear Materials Inventory and Source Data, November 1986

Monthly Nuclear Materials Inventory and Source Data, December 1986

Monthly Nuclear Materials Inventory and Source Data, January 1987

Monthly Nuclear Materials Inventory and Source Data, February 1987

Monthly Nuclear Materials Inventory and Source Data, March 1987

Monthly Nuclear Materials Inventory and Source Data, April 1987

Monthly Nuclear Materials Inventory and Source Data, May 1987

$06 / 19 / 87$
Monthly Nuclear Materials Inventory and Source Data, June 1987 


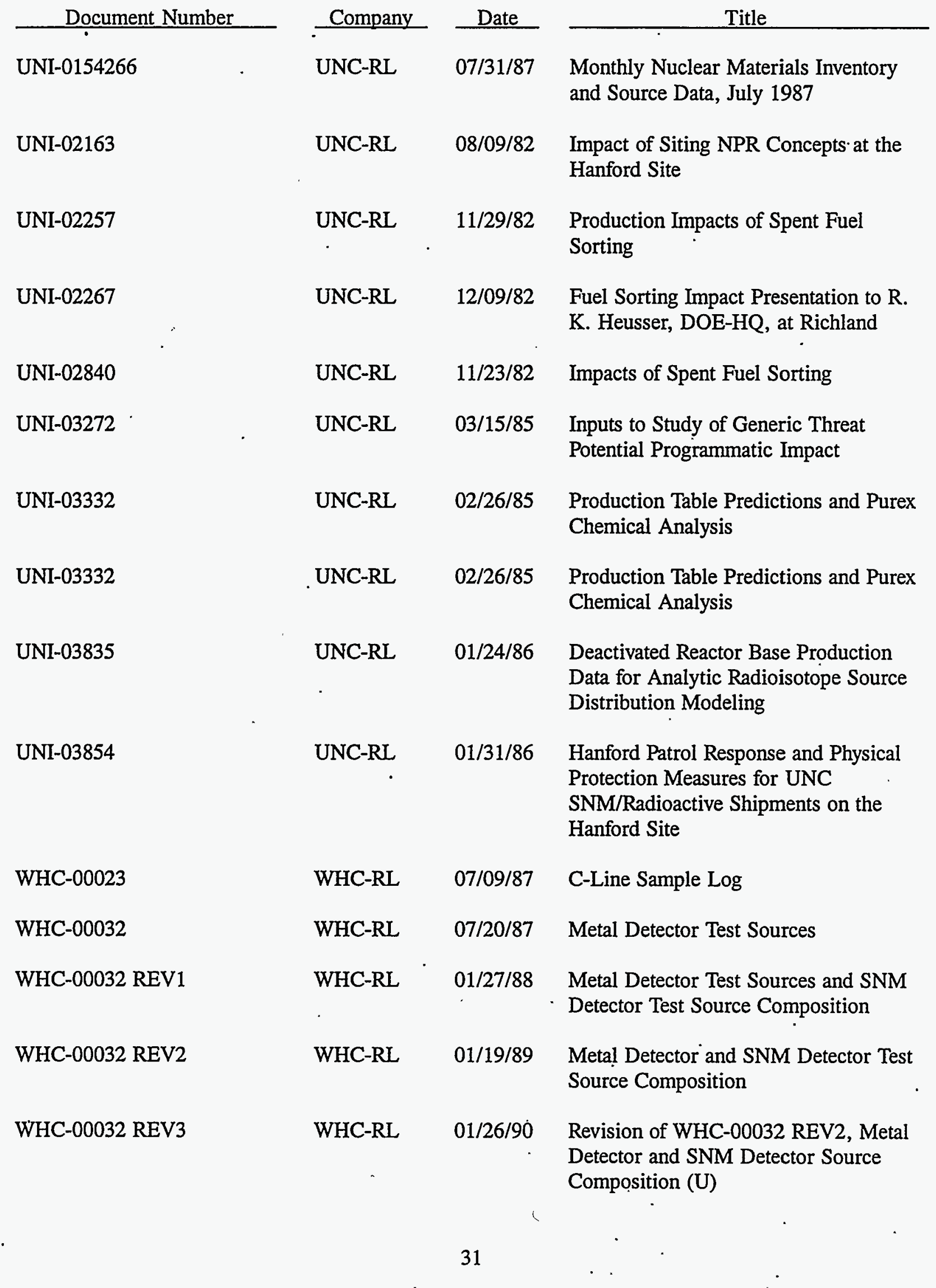


Document Number

WHC-00047 01

WHC-00047 02

WHC-00047 03

WHC-00047 04

WHC-00047 05

WHC-00093 01

WHC-00093 02

WHC-00093 03

WHC-00093 04

WHC-00093 05

WHC-00194

WHC-00247

WHC-00264
Company

WHC

WHC

WHC-RL

WHC-RL

$11 / 30 / 87$

$12 / 30 / 87$

WHC-RL

WHC-RL

WHC-RL

$11 / 12 / 87$

WHC-RL

$12 / 11 / 87$

WHC-RL $\quad 01 / 11 / 88$

WHC-RL

$10 / 15 / 87$

WHC-RL

$11 / 25 / 87$

$11 / 25 / 87$

Rockwell Hanford Operations Chemical Processing Monthly Report, November 1984 (RHO-PB-SR-10 B CP, November 1984)

WHC-RL 12/09/87 Impact on Plutonium Shipments of Reduced Processing Budgets After FY 88 
Document Number

WHC-00272

WHC88-00015

WHC88-00047 06

WHC88-00047 07

WHC88-00047 08

WHC88-00047 09

WHC88-00047 10 .

WHC88-00047 11

WHC88-00047 12

WHC88-00047 13

WHC88-00047 14

WHC88-00047 15 .

WHC88-00047 16

WHC88-00047 17

$\begin{array}{ll}\text { Company } & \text { Date } \\ \text { WHC-RL } & 12 / 15 / 87\end{array}$

WHC-RL : 01/11/88

01/30/87 Monthly Nuclear Materials Inventory and Source Data, January 1988

02/29/88 Monthly Nuclear Materials Inventory and Source Data, February 1988

03/31/88 Monthly Nuclear Materials Inventory and Source Data, March 1988

04/30/88 Monthly Nuclear Materials Inventory and Source Data, April 1988

05/31/88 Monthly Nuclear Materials Inventory and Source Data, May 1988

06/30/88 Monthly Nuclear Materials Inventory and Source Data, June 1988

07/31/88 Monthly Nuclear Materials Inventory and Source Data, July 1988

WHC-RL $\quad 08 / 31 / 88$

August 1988 Monthly Nuclear Materials Inventory and Source Data

WHC-RL 09/30/88 September 1988 Monthly Nuclear Materials Inventory and Source Data

WHC-RL 10/31/88 October 1988 Monthly Nuclear Materials Inventory and Source Data

WHC-RL 11/30/88 November 1988 Monthly Nuclear Materials Inventory and Source Data

WHC-RL 12/31/88 December 1988 Monthly Nuclear Materials Inventory and Source Data 


\begin{tabular}{|c|c|c|c|}
\hline Document Number & Company & Date & Title \\
\hline WHC88-00049 & WHC-RL & $02 / 01 / 88$ & $\begin{array}{l}\text { Spent Fuel Isotopic and Chemical } \\
\text { Analysis (Working papers) }\end{array}$ \\
\hline WHC88-0009301 & WHC-RL & $02 / 12 / 88$ & $\begin{array}{l}\text { Jan } 1988 \text { Westinghouse Nuclear } \\
\text { Materials Source Data }\end{array}$ \\
\hline WHC88-0009302 & WHC-RL & $03 / 11 / 88$ & $\begin{array}{l}\text { February } 1988 \text { Westinghouse Nuclear } \\
\text { Materials Source Data }\end{array}$ \\
\hline WHC88-0009303 & WHC-RL & $04 / 12 / 88$ & $\begin{array}{l}\text { March } 1988 \text { Westinghouse Nuclear } \\
\text { Materials Source Data }\end{array}$ \\
\hline WHC88-0009304 & WHC-RL & $05 / 12 / 88$ & $\begin{array}{l}\text { April } 1988 \text { Westinghouse Nuclear } \\
\text { Materials Source Data }\end{array}$ \\
\hline WHC88-0009305 & WHC-RL & $06 / 13 / 88$ & $\begin{array}{l}\text { May } 1988 \text { Westinghouse Nuclear } \\
\text { Materials Source Data }\end{array}$ \\
\hline WHC88-0009306 & WHC-RL & $07 / 12 / 88$ & $\begin{array}{l}\text { June } 1988 \text { Westinghouse Nuclear } \\
\text { Materials Source Data }\end{array}$ \\
\hline WHC88-0009307 & WHC-RL & $08 / 12 / 88$ & $\begin{array}{l}\text { July } 1988 \text { Westinghouse Nuclear } \\
\text { Materials Source Data }\end{array}$ \\
\hline WHC88-00093 08 & WHC-RL & 09/12/88 & $\begin{array}{l}\text { August } 1988 \text { Westinghouse Nuclear } \\
\text { Materials Source Data }\end{array}$ \\
\hline WHC88-0009309 & WHC-RL & $10 / 12 / 88$ & $\begin{array}{l}\text { September } 1988 \text { Westinghouse Nuclear } \\
\text { Materials Source Data }\end{array}$ \\
\hline WHC88-0009310 & WHC-RL & $11 / 11 / 88$ & $\begin{array}{l}\text { October } 1988 \text { Westinghouse Nuclear } \\
\text { Materials Source Data }\end{array}$ \\
\hline WHC88-00093 11 & WHC-RL & $12 / 12 / 88$ & $\begin{array}{l}\text { November } 1988 \text { Westinghouse Nuclear } \\
\text { Materials Source Data }\end{array}$ \\
\hline WHC88-00093 12 & WHC-RL & $01 / 12 / 89$ & $\begin{array}{l}\text { December } 1988 \text { Westinghouse Nuclear } \\
\text { Materials Source Data }\end{array}$ \\
\hline WHC88-00136 & WHC-RL & $03 / 11 / 88$ & $\begin{array}{l}\text { Rockwell Hanford Operations Chemical } \\
\text { Processing Monthly Report, November } \\
1987 \text { (RHO-PB-SR-10 B CP NOV84) }\end{array}$ \\
\hline WHC88-00159 & WHC-RL & $03 / 31 / 88$ & $\begin{array}{l}\text { Chemical Processing Historical } \\
\text { Plutonium Losses }\end{array}$ \\
\hline
\end{tabular}




\begin{tabular}{|c|c|c|c|}
\hline Document Number & Company & Date & Title \\
\hline WHC88-00159 DEL & WHC-RL & $03 / 31 / 88$ & $\begin{array}{l}\text { Chemical Processing Historical } \\
\text { Plutonium Losses }\end{array}$ \\
\hline WHC88-00185 & WHC-RL & $04 / 14 / 88$ & $\begin{array}{l}\text { Chemical Processing Historical } \\
\text { Production }\end{array}$ \\
\hline WHC88-00185 & WHC-RL & $04 / 14 / 88$ & $\begin{array}{l}\text { Chemical Processing Historical } \\
\text { Production }\end{array}$ \\
\hline WHC88-00185 DEL & WHC-RL & $04 / 14 / 88$ & $\begin{array}{l}\text { Chemical Processing Historical } \\
\text { Production }\end{array}$ \\
\hline WHC88-00200 & WHC-RL & $04 / 01 / 88$ & $\begin{array}{l}\text { Unit Cost Comparisons of RMC Line } \\
\text { and Pyrochemical Metal Production } \\
\text { Processes }\end{array}$ \\
\hline WHC88-00205 & WHC-RL & $06 / 28 / 88$ & $\begin{array}{l}\text { Classified ADP System Facility Security } \\
\text { Plan Safeguards Group - } 2704 Z \\
\text { Building Classified Stand-Alone } \\
\text { Personal Computing Resources }\end{array}$ \\
\hline WHC88-00205 REV1 & WHC-RL & $10 / 31 / 88$ & $\begin{array}{l}\text { Classified ADP System Facility Security } \\
\text { Plan Safeguards Group } 2704 Z \text { Bldg } \\
\text { Classified Stand Alone Personal } \\
\text { Computing Resources }\end{array}$ \\
\hline WHC88-00222 PT3 & WHC-RL & $01 / 09 / 89$ & $\begin{array}{l}\text { Porthsmouth Gaseous Diffusion Plant } \\
\text { Resource Plan }\end{array}$ \\
\hline WHC88-00263 & WHC-RL & $07 / 19 / 88$ & 227-S (Load-In Samples) \\
\hline WHC88-00264 & WHC-RL & $07 / 19 / 88$ & RMC Samples-Metal \\
\hline WHC88-00305 & WHC-RL & $08 / 16 / 88$ & Unattended Sealed Door Source \\
\hline WHC88-00325 & WHC-RL & $09 / 29 / 88$ & $\begin{array}{l}\text { Chemical Processing FY } 1988 \text { Year-End } \\
\text { Review }\end{array}$ \\
\hline WHC88-00325 VG & WHC & $09 / 22 / 88$ & $\begin{array}{l}\text { Chemical Processing FY } 1988 \text { Year End } \\
\text { Review }\end{array}$ \\
\hline WHC88-00355 & WHC-RL & $10 / 28 / 88$ & $\begin{array}{l}\text { Nuclear Materials Source } \\
\text { Documentation (continued from RHO- } \\
\text { PO-NB-5) }\end{array}$ \\
\hline
\end{tabular}


Document Number

WHC88-00355 BK1

WHC88-00396

WHC88-00396

WHC89-00047 01

WHC89-00047 02

WHC89-00047 03

WHC89-00047 04

WHC89-00047 05

WHC89-00047 06

WHC89-00047 07

WHC89-00047 08

WHC89-00047 09

WHC89-00047 10

WHC89-00047 11

$\begin{array}{cc}\text { Company } & \text { Date } \\ \text { WHC-RL } & 08 / 04 / 89\end{array}$

WHC-RL

$12 / 05 / 88$

WHC-RL

$12 / 05 / 88$

WHC-RL

$01 / 31 / 89$

WHC-RL $\quad 02 / 28 / 89$

WHC-RL

$03 / 31 / 89$

WHC-RL

04/30/89

WHC-RL

$05 / 31 / 89$

WHC-RL

$06 / 30 / 89$

WHC-RL $\quad 07 / 31 / 89$

WHC-RL

$08 / 31 / 89$

WHC-RL

09/30/89

WHC-RL

$10 / 31 / 89$

WHC-RL

$11 / 30 / 89$
Title

Nuclear Materials Source

Documentation (continued from WHC88-00355)

DOE Order Review Summary of WHC Comments, Impacts and Implementation Plans, December 5, 1988

DOE Order Review Summary of WHC Comments, Impacts and Implementation Plans, December 5, 1988

Monthly Nuclear Materials Inventory and Source Data, January 1989.

Monthly Nuclear Materials Inventory and Source Data, February 1989

Monthly Nuclear Materials Inventory and Source Data, March 1989

- Monthly Nuclear Materials Inventory and Source Data, April 1989

Monthly Nuclear Materials Inventory and Source Data, May 1989

Monthly Nuclear Materials Inventory and Source Data, June 1989

Monthly Nuclear Materials Inventory and Source Data, July 1989

Monthly Nuclear Materials Inventory and Source Data, August 1989

Monthly Nuclear Materials Inventory and Source Data, September 1989

Monthly Nuclear Materials Inventory and Source Data, October 1989

Monthly Nuclear Materials Inventory and Source Data, November 1989 


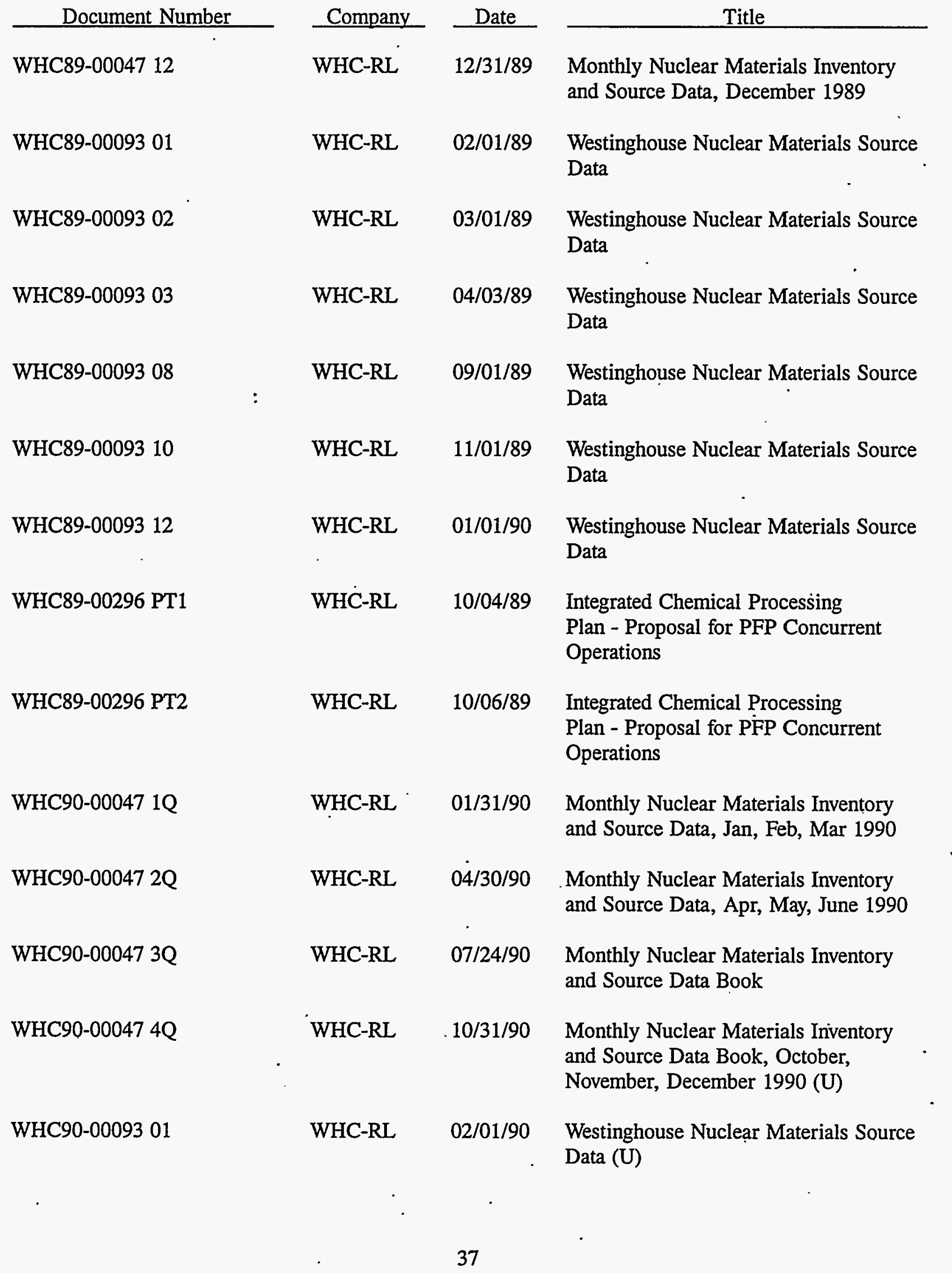




\begin{tabular}{|c|c|c|c|}
\hline Document Number & Company & Date & Title \\
\hline WHC90-00093 02 & WHC-RL & $03 / 01 / 90$ & $\begin{array}{l}\text { Westinghouse Nuclear Materials Source } \\
\text { Data (U) }\end{array}$ \\
\hline WHC90-0009303 & WHC-RL & $03 / 01 / 90$ & $\begin{array}{l}\text { Westinghouse Nuclear Materials Source } \\
\text { Data (U) }\end{array}$ \\
\hline WHC90-0009304 & WHC-RL & $03 / 01 / 90$ & $\begin{array}{l}\text { Westinghouse Nuclear Materials Source } \\
\text { Data (U) }\end{array}$ \\
\hline WHC90-0009305 & WHC-RL & $03 / 01 / 90$ & $\begin{array}{l}\text { Westinghouse Nuclear Materials Source } \\
\text { Data (U) }\end{array}$ \\
\hline WHC90-0009306 & WHC-RL & $03 / 01 / 90$ & $\begin{array}{l}\text { Westinghouse Nuclear Materials Source } \\
\text { Data (U) }\end{array}$ \\
\hline WHC90-00093 07 & WHC-RL & $03 / 01 / 90$ & $\begin{array}{l}\text { Westinghouse Nuclear Materials Source } \\
\text { Data (U) }\end{array}$ \\
\hline WHC90-0009308 & WHC-RL & $09 / 01 / 90$ & $\begin{array}{l}\text { Westinghouse Nuclear Materials Source } \\
\text { Data (U) }\end{array}$ \\
\hline WHC90-0009309 & WHC-RL & $10 / 01 / 90$ & $\begin{array}{l}\text { Westinghouse Nuclear Materials Source. } \\
\text { Data (U). }\end{array}$ \\
\hline WHC90-0009310 & WHC-RL & $11 / 01 / 90$ & $\begin{array}{l}\text { Westinghouse Nuclear Materials Source } \\
\text { Data (U) }\end{array}$ \\
\hline WHC90-00093 11 & WHC-RL & $12 / 01 / 90$ & $\begin{array}{l}\text { Westinghouse Nuclear Materials Source } \\
\text { Data (U) }\end{array}$ \\
\hline WHC90-00093 12 & WHC-RL & $01 / 01 / 91$ & $\begin{array}{l}\text { Westinghouse Nuclear Materials Source } \\
\text { Data }(U)\end{array}$ \\
\hline WHC91-00035 & WHC-RL & $02 / 12 / 91$ & $\begin{array}{l}\text { Remote Mechanical "C" Line Sample } \\
\text { Analysis (U) }\end{array}$ \\
\hline WHC91-00043 & WHC-RL & $02 / 27 / 91$ & PGDP Resource Plan (U) \\
\hline WHC91-00047 1Q & WHC-RL & $01 / 16 / 91$ & $\begin{array}{l}\text { Monthly Nuclear Materials Inventory } \\
\text { and Source Data Book for January, } \\
\text { February, and March } 1991 \text { (U) }\end{array}$ \\
\hline WHC91-00047 2Q & WHC-RL & $05 / 08 / 91$ & $\begin{array}{l}\text { Monthly Nuclear Material Inventory and } \\
\text { Source Data Book for April, May, and } \\
\text { June } 1991 \text { (U) }\end{array}$ \\
\hline
\end{tabular}


Document Number

WHC91-00047 3Q

WHC91-00047 4Q

WHC91-00093 01

WHC91-00093 02

WHC91-00093 03

WHC91-00093 04

WHC91-00093 05.

WHC91-00093 06

WHC91-0009307

WHC91-0009308

WHC91-0009309

WHC91-0009310

WHC91-00093 11

WHC91-00093 12

WHC91-00104

$\begin{array}{ll}\text { Company } & \text { Date } \\ \text { WHC-RL } & 06 / 25 / 91\end{array}$

Monthly Nuclear Materials Inventory and Source Data Book for July, August, and September (U)

WHC-RL 10/04/91 Monthly Nuclear Materials Inventory and Source Data Book for October (U)

WHC-RL 01/01/91 Westinghouse Nuclear Máterials Source Data (U)

WHC-RL 03/01/91 Westinghouse Nuclear Materials Source Data (U)

WHC-RL 04/01/91 Westinghouse Nuclear Materials Source Data (U)

WHC-RL 05/01/91 Westinghouse Nuclear Materials Source Data (U)

WHC-RL 06/01/91 Westinghouse Nuclear Materials Source Data (U)

WHC-RL 07/01/91 Westinghouse Nuclear Materials Source Data (U)

WHC-RL 08/01/91 Westinghouse Nuclear Materials Source Data (U)

WHC-RL 09/01/91 Westinghouse Nuclear Materials Source Data (U)

WHC-RL 10/01/91 Westinghouse Nuclear Materials Source Data (U)

WHC-RL 11/01/91 Westinghouse Nuclear Materials Source Data (U)

W.HC-RL 12/01/91 Westinghouse Nuclear Materials Source Data (U)

WHC-RL 01/01/92 Westinghouse Nuclear Materials Source Data (U)

WHC-RL 05/29/91 MBA 221 Source Document Forms (U) 


\begin{tabular}{|c|c|c|c|}
\hline Document Number & Company & Date & Title \\
\hline WHC91-00142DR & WHC-RL & $08 / 27 / 91$ & $\begin{array}{l}\text { Hanford Nuclear Material Item } \\
\text { Inventory Numbers for NTRAK } \\
\text { Detector Test SNM Sources (U) }\end{array}$ \\
\hline WHC92-00034 & WHC-RL & $08 / 27 / 92$ & $\begin{array}{l}\text { KARE-I SNM Specimen Description } \\
\text { and Thermal Analysis (U) }\end{array}$ \\
\hline WHC92-00034DR & WHC-RL & $08 / 21 / 92$ & $\begin{array}{l}\text { KARE-I SNM Specimen Description } \\
\text { and Thermal Analysis (U) }\end{array}$ \\
\hline WHC92-00036 & WHC-RL & $08 / 19 / 92$ & $\begin{array}{l}\text { KARE-1 SNM Subcapsule Tempest } \\
\text { Thermal Analysis Input Decks and } \\
\text { Results (U) }\end{array}$ \\
\hline WHC92-0009301 & WHC-RL & $02 / 03 / 92$ & $\begin{array}{l}\text { Westinghouse Nuclear Materials Source } \\
\text { Data (U) }\end{array}$ \\
\hline WHC92-0009302 & WHC-RL & $03 / 02 / 92$ & $\begin{array}{l}\text { Westinghouse Nuclear Materials Source } \\
\text { Data (U) }\end{array}$ \\
\hline WHC92-00093 03 & WHC-RL & $04 / 01 / 92$ & $\begin{array}{l}\text { Westinghouse Nuclear Materials Source } \\
\text { Data (U) }\end{array}$ \\
\hline WHC92-0009304 & WHC-RL & $05 / 01 / 92$ & $\begin{array}{l}\text { Westinghouse Nuclear Material Source } \\
\text { Data (U) }\end{array}$ \\
\hline WHC92-0009305 & WHC-RL & $06 / 01 / 92$ & $\begin{array}{l}\text { Westinghouse Nuclear Material Source } \\
\text { Data (U) }\end{array}$ \\
\hline WHC92-0009306 & WHC-RL & $06 / 01 / 92$ & $\begin{array}{l}\text { Westinghouse Nuclear Material Source } \\
\text { Data (U) }\end{array}$ \\
\hline WHC92-00093 07 & WHC-RL & 08/01/92 & $\begin{array}{l}\text { Westinghouse Nuclear Material Source } \\
\text { Data (U) }\end{array}$ \\
\hline WHC92-0009308 & WHC-RL & 09/01/92 & $\begin{array}{l}\text { Westinghouse Nuclear Material Source } \\
\text { Data (U) }\end{array}$ \\
\hline WHC92-00093 09 & WHC-RL & $10 / 01 / 92$ & $\begin{array}{l}\text { Westinghouse Nuclear Material Source } \\
\text { Data (U) }\end{array}$ \\
\hline WHC92-0009310 & WHC-RL & $11 / 01 / 92$ & $\begin{array}{l}\text { Westinghouse Nuclear Material Source } \\
\text { Data (U) }\end{array}$ \\
\hline
\end{tabular}


Document Number

WHC92-00093 11

WHC92-00093 12

WHC93-00009 02

WHC93-00009 03

WHC93-00093 01

WHC93-00093 02

WHC93-00093 03

WHC93-00093 04

WHC93-00093 05

WHC93-00093 06

WHC93-00093 07

WHC93-0009308

WHC93-00093 09

WHC93-0009310

WHC93-00093 11

$\frac{\text { Company }}{\text { WHC-RL }} \frac{\text { Date }}{12 / 01 / 92}$

WHC-RL 01/01/93

$$
\text { . }
$$

WHC-RL 03/01/93

.

WHC-RL 04/01/93

WHC-RL 02/01/93

WHC-RL . 03/01/93

WHC-RL 04/01/93

Westinghouse Nuclear Material Source
Data (U)

WHC-RL 05/01/93 Westinghouse Nuclear Material Source Data (U)

WHC-RL 06/01/93 Westinghouse Nuclear Material Source Data (U)

WHC-RL 07/01/93 Westinghouse Nuclear Material Source Data (U)

WHC-RL 08/01/93 Westinghouse Nuclear Material Source Data (U)

WHC-RL 09/01/93 Westinghouse Nuclear Material Source Data (U)

WHC-RL 10/01/93 Westinghouse Nuclear Material Source Data (U) .

WHC-RL 11/01/93 Westinghouse Nuclear Material Source Data (U)

WHC-RL 12/01/93 Westinghouse Nuclear Material Source Data (U) 
Document Number

WHC93-00093 12

Z86.1035

Z86.57 DRAFT
Company Date

WHC-RL 01/01/93

Westinghouse Nuclear Material Source

Data (U)

DOE-HQ 11/01/81 Study of the Impacts of Draft Threat Guidance Order (5600) Upon the Richland Operations Office

DOE-HQ
Malevolent Dispersal of Radioactive

Materials: An Information Assessment 


\section{Distribution}

No. of

Copies

Offsite

12 DOE Office of Scientific and Technical Information

M. Bauer

Confederated Tribes and Bands of the Yakama Indian Nations

P.O. Box 151

Toppenish, WA 98948

S. Benz

600 S. Kent \#71

Kennewick, WA 99336

L. Block

U.S. Fish and Wildlife Service Moses Lake Field Office

P.O. Box 1157

Moses Lake, WA 98837

D. Bradshaw

Audobon Society of Portland

5151 Northwest Cornell Road

Portland, OR 97210

N. Buske

Search Technical Services

Star Route Box 17

Davenport, WA 99112

D. Cellarius

Sierra Club Northwest Office

1516 Melrose Avenue

Seattle, WA 98122

C. Cline

Washington State Dept. of Ecology'

Mail Stop PV-11

P.O. Box 47651

Olympia, WA 98504-8711
No. of

Copies

F. R. Cook

2552 Harris

Richland, WA 99352

J. P. Corley

2213 Torbett

Richland, WA 99352

2 DNR Hanford Projects

Attn: J. R. Wilkinson

Confederated Tribes of the Umatilla Indian Nation

P.O. Box 638

Pendleton, OR 97801

G. deBruler

Columbia River United

P.O. Box 667

Bingen, WA 98605

C. Denniston

Greenpeace

11815 - 20th SW

Seattle, WA 98146

B. Drost

USGS Water Resources Division

Washington District

1201 Pacific Avenue Suite 600

Tacoma, WA 98402

D. Dunning

Oregon Dept. of Energy

625 Marion Street NE

Salem, OR 97310

3 J. Erickson

Division of Radiation Protection

Washington State Dept. of Health

P.O. Box 47827

Olympia, WA 98504-7827

Distr. 1 
No. of

Copies

M. Fox, President

Hanford Family

P.O. Box 1462

Richland, WA 99352

R. Gạdiner

614 Sherman Drive

The Dalles, OR 97058

N. J. Germond

League of Women Voters

224 Iron Mountain Boulevard

Lake Oswego, OR 97034

D. Maez

4214 W. John Day Place

Kennewick, WA 99336

C. Mebane

National Oceanic and Atmospheric

Administration

c/o EPA Seattle Office

1200 6th Avenue, HW-113

Seattle, WA 98101

J. Monteith

Oregon Natural Resources Council 1161 Lincoln Street

Eugene, OR 97401

D. Nichols

WNP-1

Building 52

Richland, WA 99352

R. Patt

Water Resources Department

555 13th Street, NE

Salem, OR 97301

G. Pollet

Heart of America Northwest

1305 4th Avenue, 208

Seattle, WA 98101
No. of

Copies

2 D. Powaukee

Nez Perce Tribe

P.O. Box 365

Lapwai, ID 83540

L. Stembridge

HEAL

1408 West Broadway Avenue

Spokane, WA 99201-1902

C. Stephan

1520 Torthay Court

Richland, WA 99352

3 D. Stewart-Smith

Oregon Department of Energy

625 Marion Street NE

Salem, OR 97310

J. P. Thomas

HHIN Resource Center

1719 Smith Tower

506 Second Avenue

Seattle, WA 98104

Onsite

11 DOE Richland Operations Office

R. F. Brich (5)

H4-83

P. W. Willison

A4-52

DOE Public Reading Room (5) H2-53

6 Washington State Department of Ecology

S. M. Alexander

B5-18

D. P. Holland (4)

B5-18

J. W. Yokel

B5-18

4 Westinghouse Hanford Company

Administrative Record (3)

H6-08

A. S. Carlson

B3-35

Distr. 2 
No. of

Copies

6 U. S. Environmental Protection Agency

L. E. Gadbois (5) B5-01

D. R. Sherwood B B-01

34 Pacific Northwest Laboratory
S. D. Cannon
R. L. Dirkes
P. W. Eslinger
S. L. Friant
M. S. Hanson
G. L. Harvey
P. 'C. Hays

No. of

Copies

L. R. Huesties

K6-61

A. D. Maughan

K6-52

K5-12

K6-62

K3-54

K3-70

K9-13

K3-54

K9-13

K9-33

K9-04

K3-70

K1-06

P8-55 